\title{
Large-scale amplitude modulation of the small-scale structures in turbulent boundary layers
}

\author{
ROMAIN MATHIS, NICHOLAS HUTCHINS \\ AND IVAN MARUSIC $†$ \\ Walter Bassett Aerodynamics Laboratory, Department of Mechanical Engineering, University of \\ Melbourne, Victoria 3010, Australia
}

(Received 7 December 2008 and in revised form 1 March 2009)

In this paper we investigate the relationship between the large- and small-scale energy-containing motions in wall turbulence. Recent studies in a high-Reynoldsnumber turbulent boundary layer (Hutchins \& Marusic, Phil. Trans. R. Soc. Lond. A, vol. 365, 2007a, pp. 647-664) have revealed a possible influence of the largescale boundary-layer motions on the small-scale near-wall cycle, akin to a pure amplitude modulation. In the present study we build upon these observations, using the Hilbert transformation applied to the spectrally filtered small-scale component of fluctuating velocity signals, in order to quantify the interaction. In addition to the large-scale log-region structures superimposing a footprint (or mean shift) on the nearwall fluctuations (Townsend, The Structure of Turbulent Shear Flow, 2nd edn., 1976, Cambridge University Press; Metzger \& Klewicki, Phys. Fluids, vol. 13, 2001, pp. 692701.), we find strong supporting evidence that the small-scale structures are subject to a high degree of amplitude modulation seemingly originating from the much larger scales that inhabit the log region. An analysis of the Reynolds number dependence reveals that the amplitude modulation effect becomes progressively stronger as the Reynolds number increases. This is demonstrated through three orders of magnitude in Reynolds number, from laboratory experiments at $R e_{\tau} \sim 10^{3}-10^{4}$ to atmospheric surface layer measurements at $R e_{\tau} \sim 10^{6}$.

\section{Introduction}

Over the past few decades, a great many studies have been directed towards understanding the turbulence structure in the near-wall region of wall-bounded flows. To a large extent, such studies have their origins in the observations of Kline et al. (1967) and the realization that recurrent near-wall structures can play a key role in turbulence regeneration. More recently our understanding of such events has tended to shift towards a self-sustaining near-wall cycle, in which the near-wall structures propagate and sustain without need of external triggers (Panton 2001). Such autonomous views are based largely on insightful low-Reynolds-number simulations by Jiménez \& Pinelli (1999) and Schoppa \& Hussain (2002).

The logarithmic region was largely absent from the earliest low-Reynoldsnumber flow visualizations and direct numerical simulation (DNS). For example, the 
approximate upper and lower bounds of the logarithmic region $\left(100<z^{+}<0.15 \delta^{+}\right)$ would indicate that almost no overlap region was present in the measurements of Kline et al. (1967) or in DNS in which $R e_{\tau} \leqslant 700$. (The friction Reynolds number or Kármán number is defined as $R e_{\tau}=\delta U_{\tau} / \nu$, where $\delta$ is the boundarylayer thickness, $U_{\tau}$ the friction velocity and $v$ the kinematic viscosity.) The first insights into the log-region structure were provided by performing spatio-temporal two-point correlations across the boundary layer (using hot wires). Statistics based on fluctuating streamwise velocity signals revealed the presence of highly elongated regions of uniform streamwise momentum in the log region (Blackwelder \& Kovasznay 1972; Wark \& Nagib 1991; Wark, Naguib \& Robinson 1991). More recently, advances in particle image velocimetry (PIV) and DNS have provided the opportunity to study the turbulence structure in the logarithmic region of higher-Reynolds-number turbulent boundary layers (Adrian, Meinhart \& Tomkins 2000; del Álamo et al. 2004; Hoyas \& Jiménez 2006). PIV studies of streamwise/spanwise planes have revealed the presence of a pronounced stripiness in instantaneous fields of streamwise velocity (u) fluctuation (Ganapathisubramani, Longmire \& Marusic 2003; Tomkins \& Adrian 2003; Hutchins \& Marusic 2005; Hambleton, Hutchins \& Marusic 2006). Similar events were observed in the channel flow numerical simulations of del Alamo \& Jiménez (2003). Such elongated regions of momentum deficit have been explained within vortex-based models as the regions between the legs of aligned packets of hairpin vortices (Adrian et al. 2000; Ganapathisubramani et al. 2003; Tomkins \& Adrian 2003; Hambleton et al. 2006; and others). These low-speed regions are typically $0.3-0.5 \delta$ wide in the spanwise direction and often seem to occur in spanwise alternating patterns. (Elongated low-speed events are usually flanked on either side by high-speed events.) The length of these features often exceeds the streamwise length of the PIV images. Hutchins \& Marusic (2007a) employed spanwise rakes of hot-wire probes to ascertain the true length of these structures, demonstrating that they appear to extend to lengths of order $20 \delta$ and meander substantially. They used the collective term 'superstructures' (so named because of their large size and because they are shown to carry a large proportion of the Reynolds shear stress) to describe these events. Ganapathisubramani, Clemens \& Dolling (2006) used a multiple sideby-side arrangement of cameras to image $8 \delta \times 2 \delta$ streamwise/spanwise planes in a supersonic turbulent boundary layer, finding similar elongated meandering features. For pipe flows, the energetic footprint of superstructure-type events is evident as even longer-wavelength peaks in the pre-multiplied energy spectra of $u$ fluctuations, termed very large-scale motions or VLSMs (Kim \& Adrian 1999; Abe, Kawamura \& Choi 2004; Toh \& Itano 2005; Guala, Hommema \& Adrian 2006). Recent studies in turbulent pipe and channel flow facilities (Monty et al. 2007; Bailey et al. 2008) have reiterated the general presence and form of these very large features in internal geometries.

It is logical then to consider what effect these very large log-region motions might have on the near-wall cycle. We do not seek here to challenge the view of self-sustaining near-wall turbulence as suggested by Jiménez \& Pinelli (1999), the evidence for which is compelling. However, we do note that the term 'autonomous' when referring to the near-wall cycle can tend to negate the influence of larger scales, which, although perhaps not strictly a prerequisite for sustenance of the near-wall cycle, may still impart an influence or modulation on near-wall events. One clear example of such an influence is in the breakdown of universal behaviour based on viscous scaling in the near-wall region. The viscous-scaled near-wall peak in the streamwise broadband intensity clearly grows in magnitude with increasing Reynolds number (Klewicki \& Falco 1990; DeGraaff \& Eaton 2000; Metzger 
et al. 2001; Metzger \& Klewicki 2001; Marusic \& Kunkel 2003; Hoyas \& Jiménez 2006; Hutchins et al. 2009). Moreover, it has been shown that such growth is due to the increase of large-scale energy imparted to the near-wall region as $R e$ increases (Metzger \& Klewicki 2001; Hutchins \& Marusic 2007a,b). Hutchins \& Marusic (2007a) have clearly shown that the footprint of large-scale superstructure events in the streamwise velocity fluctuations can extend deep into the near-wall region. This is as predicted by Townsend (1976), who noted that the near-wall region will feel wall-parallel motions due to all attached eddies with centres above that height (right across the shear layer). Thus, in the near wall, the streamwise velocity fluctuations will be the sum of the induced fluctuations from every scale that resides above (including superstructures). In this instance the large-scale energy is merely superimposed as a low-wavenumber shift onto the near wall and, by definition (since it is largely wall-parallel), will not contribute to the Reynolds shear stress.

By studying fluctuating velocity signals from hot-wire sensors in the near-wall region, Hutchins \& Marusic (2007b) recently observed that in addition to the lowwavenumber mean shift, the largest scales appear to 'amplitude modulate' the smallscale fluctuations. They noted that the large regions of streamwise momentum deficit (associated with the low-speed footprint of the superstructures) are accompanied by reduced small-scale fluctuations in the near-wall region. On the other hand, for large-scale high-momentum regions, the small-scale fluctuating component is more energetic. They also found that, away from the wall, this scenario seems to reverse with the more energetic small-scale fluctuations eventually becoming aligned with the part of the superstructure that is in momentum deficit.

Some observations concerning the contribution of outer-scale events to the near-wall cycle have previously been reported in the literature. In their study of the 'bursting' phenomena, Rao, Narasimha \& Badri Narayanan (1971) have previously observed that large outer-scaled structures were interacting with the near-wall structure. Later, Bandyopadhyay \& Hussain (1984) studied the relationship between large and small scales in numerous shear flows, including boundary layers, mixing layers, wakes and jets. By studying short-time correlation measurements (from hot-wire time-series data) between the low-frequency component and a signal similar to the envelope of the high-frequency component, they found significant coupling between scales in all flows. They also noted a reversal in coupling, occurring only across the boundary layer and the mixing layer (referring to this as a phase reversal). They found that the large and small scales, initially 'phase-locked' in the vicinity of the wall, are progressively shifted across the boundary layer to reach a $180^{\circ}$ phase difference at the edge of the log region. More recently, Hunt \& Morrison (2000) have observed that largescale log-layer events can influence the near-wall region at high Reynolds number. Based on atmospheric data, they suggested that when the Reynolds number becomes sufficiently high the large-scale eddies associated with the log layer become dominant, influencing the near-wall region and maintaining the Reynolds shear stresses. They categorized this as a 'top-down' instability, as opposed to the 'bottom-up' mechanism that is more commonly proposed from lower-Reynolds-number studies.

In this paper, we expand upon the initial observations of Hutchins \& Marusic (2007b), using the Hilbert transformation in an attempt to quantify the relationship between large-scale fluctuations and any amplitude modulation of the small-scale energy in turbulent boundary layers. The Hilbert transform is particularly well suited to the analysis of amplitude modulation interactions and has often been used in the study of geophysical and atmospheric processes (Spark \& Dutton 1972; Hristov, Friehe \& Miller 1998; Huang, Shen \& Long 1999; Ouergli 2002). However, its use in turbulence studies is far less common than say the Fourier transform. Notable 


\begin{tabular}{rlccccccc}
\hline $\boldsymbol{R} e_{\tau}$ & Facility & $\begin{array}{c}\delta \\
(\mathrm{m})\end{array}$ & $\begin{array}{c}U_{\tau} \\
\left(\mathrm{m} \mathrm{s}^{-1}\right)\end{array}$ & $\begin{array}{c}U_{\infty} \\
\left(\mathrm{m} \mathrm{s}^{-1}\right)\end{array}$ & $l^{+}$ & $\Delta T^{+}$ & $\begin{array}{c}\text { No. of } \\
\text { points }\end{array}$ & $\begin{array}{c}\text { Domain } \\
\text { of measurement }\end{array}$ \\
2800 & HRNBLWT & 0.098 & 0.442 & 11.97 & 22 & 0.53 & 53 & $z^{+}=9.09-z / \delta=4.52$ \\
3900 & HRNBLWT & 0.140 & 0.426 & 11.87 & 22 & 0.49 & 49 & $z^{+}=11.83-z / \delta=3.18$ \\
7300 & HRNBLWT & 0.326 & 0.330 & 9.82 & 22 & 0.37 & 51 & $z^{+}=5.50-z / \delta=1.36$ \\
13600 & HRNBLWT & 0.315 & 0.671 & 20.63 & 22 & 0.48 & 54 & $z^{+}=7.44-z / \delta=1.41$ \\
19000 & HRNBLWT & 0.303 & 0.960 & 30.20 & 22 & 0.59 & 53 & $z^{+}=8.71-z / \delta=1.45$ \\
650000 & SLTEST & 60 & 0.180 & - & - & 96 & 9 & $z / \delta=0.02-z / \delta=0.43$
\end{tabular}

TABLE 1. Experimental parameters for hot-wire traverses; HRNBLWT experiments were conducted with single hot-wire probe; SLTEST data were acquired with sonic anemometers. Number of points indicates the number of wall-normal positions.

$\begin{array}{ccccccccc}R e_{\tau} & \text { Facility } & \begin{array}{c}\delta \\ (\mathrm{m})\end{array} & \begin{array}{c}U_{\tau} \\ \left(\mathrm{m} \mathrm{s}^{-1}\right)\end{array} & \begin{array}{c}U_{\infty} \\ \left(\mathrm{m} \mathrm{s}^{-1}\right)\end{array} & l^{+} & T^{+} & \begin{array}{c}\text { No. of } \\ \text { points }\end{array} & \begin{array}{c}\text { Domain } \\ \text { of measurement }\end{array} \\ 7300 & \text { HRNBLWT } & 0.325 & 0.353 & 10.28 & 22 & 0.40 & 2 & z^{+}=15 \text { and } z / \delta=0.06\end{array}$

TABLE 2. Experimental parameters for two-point synchronized hot-wire measurements.

exceptions include Sreenivasan (1985) who used the analytic signal and envelope as defined via the Hilbert transform (described in §4) to characterize the intermittency of fine-scale turbulence and more recently Tardu (2008) who used the Hilbert transform in studying the instantaneous phase and amplitude of wavelet coefficients applied to fluctuating velocity signals in turbulent boundary layers.

\section{Experimental set-up}

The experiments were conducted in the High Reynolds Number Boundary Layer Wind-Tunnel (HRNBLWT) at the University of Melbourne with a working section of dimensions $27 \mathrm{~m} \times 2 \mathrm{~m} \times 1 \mathrm{~m}$. Full details of the facility are available in Nickels et al. (2005). Measurements consist of boundary-layer traverses using a single-normal hot-wire probe. The probe is operated in constant temperature mode, using an AA Lab Systems AN-1003 with overheat ratio set to 1.8. Wollaston wires of various core diameters are soldered across the tips of the probe and etched to give a platinum filament of the desired length. For each Reynolds number, the diameter $d$ and length $l$ of the sensing element was adjusted to give a constant viscous scaled length of $l^{+}=l U_{\tau} / v=22$ with $l / d \geqslant 200$, to allow comparison without any spatial resolution influences (see Hutchins et al. 2009 for a full description of the matched $l^{+}$data set). The non-dimensional time interval between samples was maintained in the range $\Delta T^{+} \simeq 0.4-0.6$ to ensure that the smallest scales were adequately resolved. A sufficiently long sample length $T$, in the range 5000-14000 boundary-layer turnover times $\left(T U_{\infty} / \delta\right.$, where $U_{\infty}$ is the free-stream velocity), was used to converge the energy contained in the largest scales. Details of the experimental conditions are given in table 1 . The friction velocity $U_{\tau}$ was calculated from a Clauser chart fit (using loglaw constants $\kappa=0.41$ and $A=5.0$ ). Boundary-layer thickness $\delta$ is calculated from a modified Coles law of the wake fit (Jones, Marusic \& Perry 2001).

In addition to the boundary-layer traverses, two-point simultaneous measurements were performed using two hot-wire probes located at $z^{+}=15$ and $z / \delta=0.06$ for a Reynolds number $R e_{\tau}=7300$. Details are given in table 2 . 
The highest Reynolds-number measurements are atmospheric surface layer (ASL) data from the Surface Layer Turbulence and Environmental Science Test Facility (SLTEST) facility, located in the Great Salt Lake Desert in Western Utah. Full details of the facility are available in Klewicki et al. (1995), Metzger \& Klewicki (2001) and Kunkel \& Marusic (2006). The unique geography of this site allows us to obtain measurements in extremely high-Reynolds-number turbulent boundary layers $\left(R e_{\tau} \sim O\left(10^{6}\right)\right)$. The boundary layer develops naturally over $100 \mathrm{~km}$ of salt playa, which is remarkably flat and has low surface roughness. Measurements were performed using a wall-normal array of nine sonic anemometers (Campbell Scientific CSAT3) spaced logarithmically from $z=1.4 \mathrm{~m}$ to $z=25.7 \mathrm{~m}$. Experimental parameters are reported in table 1, and full details are available in Hutchins \& Marusic (2007a). We will consider here $1 \mathrm{~h}$ of data taken from a period of prolonged neutral buoyancy and steady wind conditions. In this case it is difficult to accurately estimate the boundary-layer turnover time. However, a crude estimate based on sample length of $1 \mathrm{~h}, \delta \approx 70 \mathrm{~m}$ and $U_{\infty}$ based on the mean velocity at the highest wall-normal position would indicate $T U_{\infty} / \delta$ of approximately 350 . Thus the sample length for the atmospheric measurements is rather short as compared to the laboratory data. The very long superstructure-type events in the ASL (which can be $>10 \delta$ in length) would take several minutes to advect past the measurement array. Hence, the $1 \mathrm{~h}$ of data contains only limited occurrences of these events, and as such we would expect the largest wavelengths to be improperly converged. However, in spite of this uncertainty the mean statistics from SLTEST have been found to compare well with canonical turbulent boundary layers from laboratory facilities (Hutchins \& Marusic 2007a; Marusic \& Hutchins 2008). Moreover, conditional averaging and two-point correlations of the streamwise velocity fluctuation also agree very well with laboratory results, indicating that the large scales in the ASL are remarkably similar to those observed in laboratory turbulent boundary layers (Hutchins \& Marusic 2007a; Marusic \& Hutchins 2008). Clearly the wake region of the ASL would be expected to be different from that of a laboratory boundary layer. However, such differences are also true for internal geometries (turbulent pipe and channel flow facilities, both of which have turbulent cores), and yet the work of Monty et al. (2007) has shown that the general qualitative form of the large-scale features in pipes and channel are very similar to flat-plate boundary layers (with differences in spanwise scale). This would seem to suggest that the largest scales are a relatively robust feature of wall-bounded turbulence and are only weakly affected by changes in the geometry of the facility. Hence the attempt here to use the ASL case as a representation of high-Reynolds-number behaviour would seem to be reasonable.

Throughout this paper, the coordinates $x, y$ and $z$ refer to the streamwise, spanwise and wall-normal directions. The respective fluctuating velocity components are denoted by $u, v$ and $w$. The spectral density function of the streamwise velocity fluctuation is described by $\phi_{u u}$, and the streamwise wavenumber and wavelength are denoted by $k_{x}$ and $\lambda_{x}$ respectively (where $\lambda_{x}=2 \pi / k_{x}$ ). Overbars indicate time-averaged values, and the superscript ' + ' is used to denote viscous scaling of length $z^{+}=z U_{\tau} / \nu$, velocities $u^{+}=u / U_{\tau}$ and time $t^{+}=t U_{\tau}^{2} / \nu$.

\section{Initial observations of Hutchins \& Marusic (2007b)}

The observations of Hutchins \& Marusic (2007b) were performed at a Reynolds number of $R e_{\tau}=7300$, studying the impact of 'superstructure' events on the near-wall turbulent flow organization. Measurements consisted of a boundary-layer traverse, 


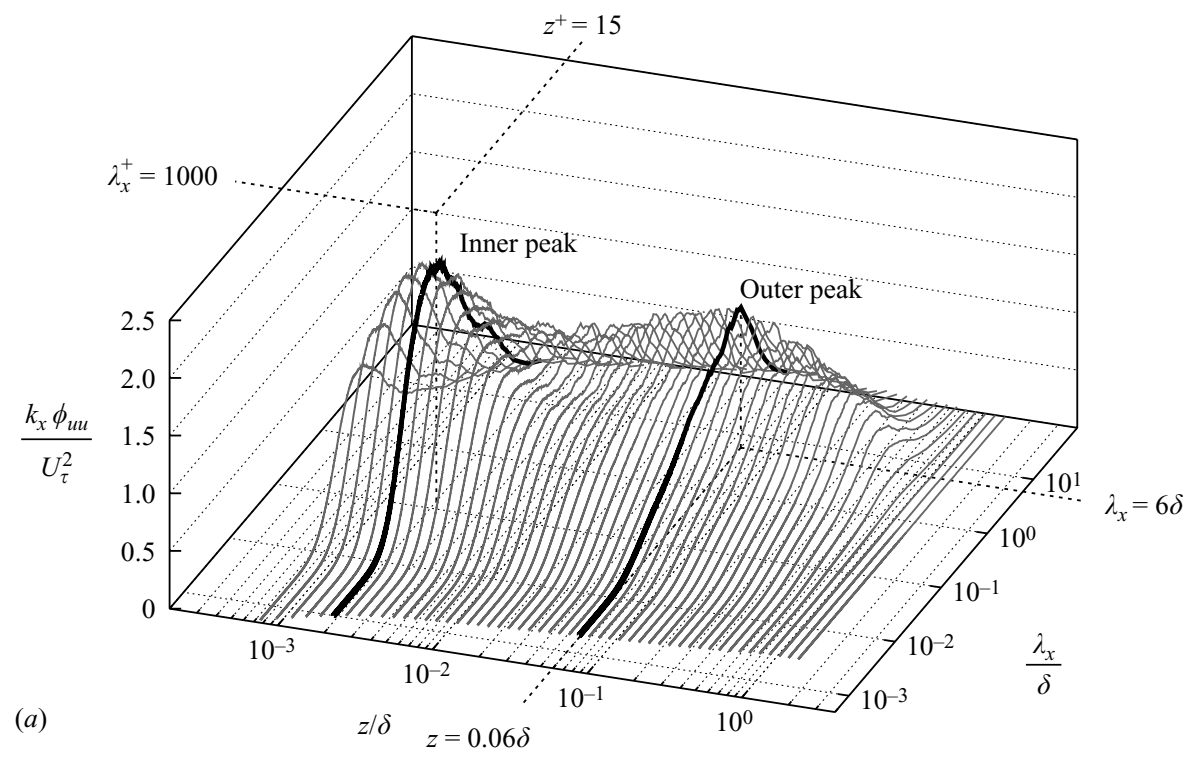

$z / \delta$

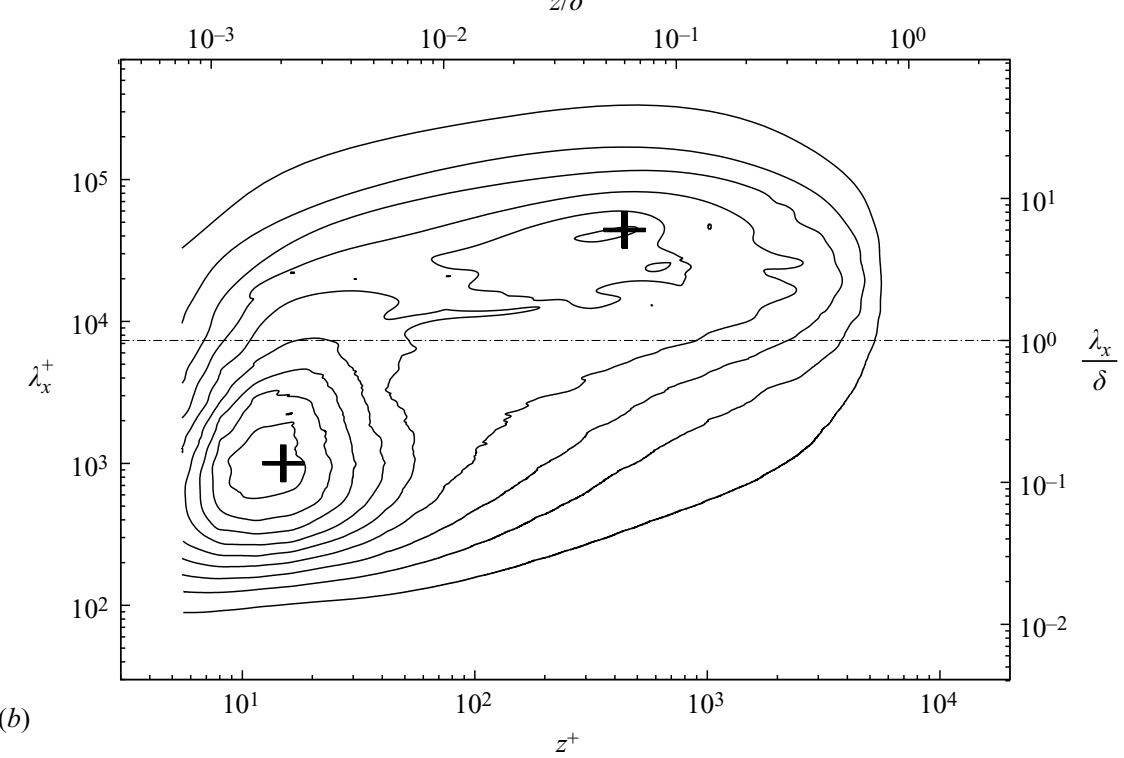

FIGURE 1. Pre-multiplied energy spectra of streamwise velocity fluctuation $k_{x} \phi_{u u} / U_{\tau}^{2}$ $\left(R e_{\tau}=7300\right)$. (a) Three dimensional surface plot for all wall-normal locations and (b) isocontour representation in which levels are from 0.2 to 1.8 in steps of 0.2 ; the ' + ' symbols mark the inner $\left(z^{+}=15, \lambda_{x}^{+}=1000\right)$ and outer $\left(z / \delta=0.06, \lambda_{x} / \delta=6\right)$ peaks. The horizontal dot-dashed line in $(b)$ shows the location of the spectral filter $\left(\lambda_{x}=\delta\right)$.

using a single normal hot-wire probe. A complete description of the experimental set-up and measurement procedure is available in Hutchins \& Marusic $(2007 a, b)$.

\subsection{Overview of the pre-multiplied energy spectra}

Following Hutchins \& Marusic (2007b), figure 1 gives an overview of the premultiplied streamwise energy spectra $k_{x} \phi_{u u} / U_{\tau}^{2}$ across the full height of the turbulent 


$\begin{array}{ccc}\text { Subscript } & \text { Designation } & \text { Spatial filter cutoff } \\ \text { L } & \text { large scales only } & \lambda_{x} / \delta>1 \\ \text { S } & \text { small scales } & \lambda_{x} / \delta<1\end{array}$

TABLE 3. Definition of filter parameters for scale decomposition.

boundary layer. Figure 1(a) shows a spectrogram for all wall-normal locations, where each profile corresponds to the one-dimensional pre-multiplied energy spectra at each wall-normal measurement station. Figure $1(b)$ is a contour view of the spectral surface shown in figure $1(a)$. It is worth noting that the representation here in terms of streamwise length scale $\left(\lambda_{x} / \delta\right)$ is only a reflected mirror image of the conventional $k_{x} \phi_{u u} / U_{\tau}^{2}$ versus $\log \left(k_{x} \delta\right)$ plot. (Equal areas under the curve will still denote equal energy.)

Two distinct peaks can be clearly observed in figure 1. (Their locations are marked by the ' + ' symbols in figure $1 b$, corresponding to the bold-lined one-dimensional profiles in figure 1a). The first peak, located in the near-wall region, is the energetic signature due to the viscous-scaled near-wall cycle of elongated high- and low-speed streaks (Kline et al. 1967). The location of this peak is fixed in viscous coordinates: $z^{+}=15$ and $\lambda_{x}^{+}=1000$. We will refer to this peak as the 'inner peak' in accordance with Hutchins \& Marusic (2007a). A second distinct peak appears in the logarithmic region. We will refer to this peak as the 'outer peak'. Initial observations of Hutchins \& Marusic (2007a) suggest that the location of this peak scales in boundary-layer thickness: $z / \delta=0.06$ and $\lambda_{x}=6 \delta$. It is of interest to note that this peak is not visible at low Reynolds numbers (where $R e_{\tau} \lesssim 1700$; see Hutchins \& Marusic 2007b) due to insufficient separation of scales and a diminishing strength at low $R e$. This outer peak is most likely the energetic signature due to the superstructure-type events (or VLSM). It has been shown (Hutchins \& Marusic 2007a) that the magnitude of this peak (when $k_{x} \phi_{u u}$ is scaled with $U_{\tau}$ ) increases with Reynolds number.

\subsection{Scale decomposition}

Using a decomposition for scales below and above a cutoff length scale, some interesting features of the signal are observed. The decomposition used here is based on a cutoff wavelength which is carefully selected from the pre-multiplied energyspectra map. The dot-dashed line in figure $1(b)$ shows the location of the cutoff, and filter parameters are given in table 3 . The cutoff $\lambda_{x} / \delta=1$ appears from the map to be a reasonable location to separate the large- (above) and small-scale (below) components of the fluctuating $u$ signal.

Figure 2 shows the decomposition of a typical fluctuating signal $u^{+}$at wall-normal position $z^{+}=15$ (inner peak). The original signal, shown in figure $2(a)$, is divided into two sub-signal components:

(i) the large-scale component $u_{L}^{+}$which is assumed to be the signature of superstructure-type events (where $\lambda_{x} / \delta>1$; figure $2 b$ );

(ii) the small-scale component signal $u_{S}^{+}$(where $\lambda_{x} / \delta<1$; figure $2 c$ );

For the large-scale component shown in figure $2(b)$, a prolonged region of negative fluctuation occurs (between the dashed lines), typical of the footprint caused by the decelerated portion of a superstructure-type event (Hutchins \& Marusic 2007a). It appears that when this negative large-scale excursion occurs, the amplitude of the small-scale fluctuations $u_{S}^{+}$is significantly reduced (figure $2 c$ ). When similar analysis is conducted for a positive large-scale excursion, the opposite scenario is true, and 

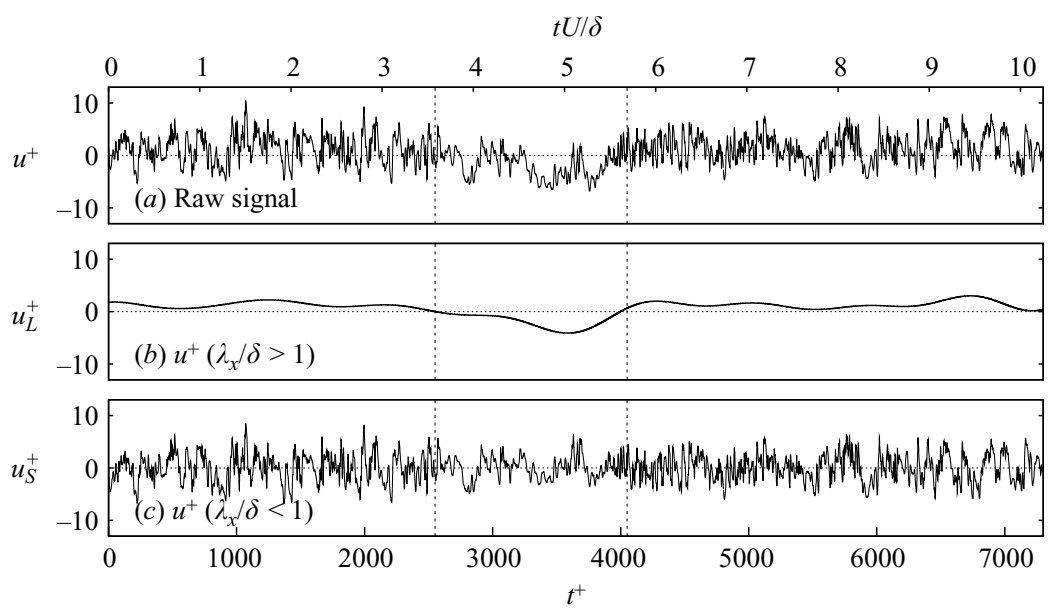

FIGURE 2. Example of fluctuating $u$ signal in the near-wall region, $z^{+}=15 ;(a)$ raw fluctuating component; $(b)$ large-scale fluctuation $\lambda_{x} / \delta>1$; (c) small-scale fluctuation $\lambda_{x} / \delta<1$. Dashed vertical lines show region of negative large-scale fluctuation.

the amplitude of the small-scale fluctuations is increased. These results suggest that the low-wavenumber motions associated with the footprints of superstructure-type events influence the near-wall $u$ fluctuations in a manner akin to a pure amplitude modulation. It should be noted that the definition of 'superstructure-type' events is based on that given by Hutchins \& Marusic 2007a, namely a spanwise alternating sequence of highly elongated negative and positive streamwise velocity fluctuations that are centred in the log region. Thus, in the proceeding analysis we consider the amplitude modulation due to both positive and negative large-scale excursions.

Based on these initial observations, a careful analysis will be conducted in the following sections using the Hilbert transform in order to define and properly understand these amplitude modulation effects.

\section{Hilbert transformation and amplitude modulation}

The Hilbert transform is a tool which has several interesting features for studying nonlinear processes. For an arbitrary real-valued signal $x(t)$, the Hilbert transformation of $x(t)$, denoted $\mathscr{X}(t)$, is given by

$$
\mathscr{X}(t)=\mathscr{H}\{x(t)\}=\frac{1}{\pi} P \int_{-\infty}^{+\infty} \frac{x(\tau)}{t-\tau} \mathrm{d} \tau,
$$

where $P$ indicates the Cauchy principal value of the integral and $\tau$ a time shift. Thus $\mathscr{X}(t)$ is the convolution integral of $x(t)$ and $(1 / \pi t)$, written as

$$
\mathscr{X}(t)=x(t) *(1 / \pi t) \text {. }
$$

From the convolution theorem, in which the Fourier transform of $(1 / \pi t)$ is given by

$$
\mathscr{H}\left\{\frac{1}{\pi t}\right\}=-\mathrm{i} \operatorname{sgn}(f)=\left\{\begin{array}{c}
-\mathrm{i} \text { for } f>0 \\
\mathrm{i} \text { for } f<0 .
\end{array}\right.
$$

It is easy to show that $\mathscr{X}(t)$ is the original signal $x(t)$ with each sinusoidal Fourier components shifted by $-90^{\circ}$ for positive frequencies and by $+90^{\circ}$ for each negative frequency. With this definition, $x(t)$ and $\mathscr{X}(t)$ form a harmonic conjugate pair, defining 


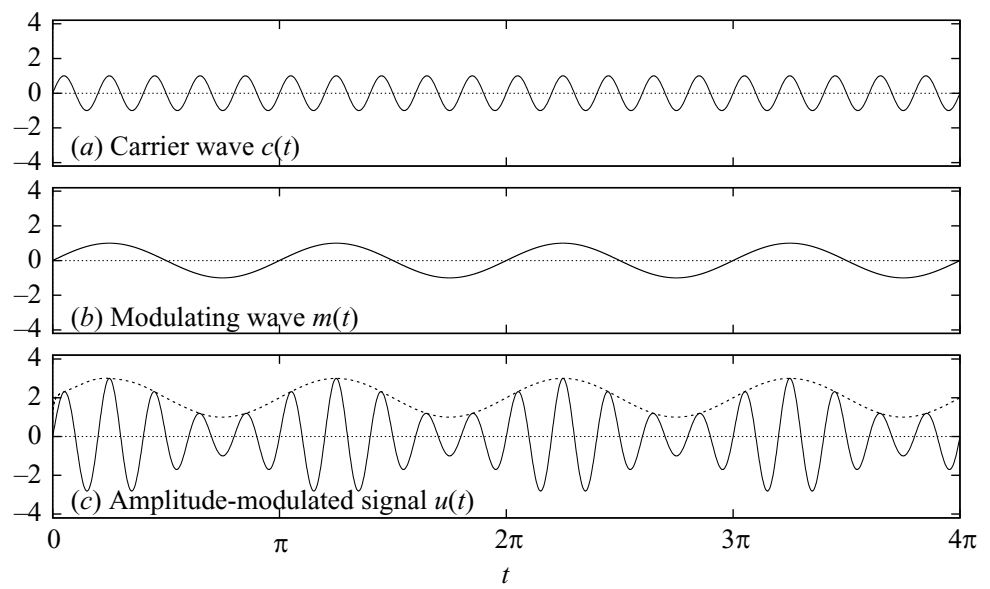

FiguRE 3. Example of amplitude modulation: $(a)$ represents the carrier wave $c(t)=\sin (10 t)$; (b) represents the modulating wave $m(t)=\sin (2 t)$; $(c)$ represents the modulated signal $u(t)=[2+m(t)] c(t)$ (solid line) and its envelope calculated from the Hilbert transform (dashed line).

the associated complex analytic signal $Z(t)$ of $x(t)$ as

$$
Z(t)=x(t)+\mathrm{i} \mathscr{X}(t)=A(t) \mathrm{e}^{\mathrm{i} \phi(t)},
$$

where $A(t)$ and $\phi(t)$ indicate instantaneous modulus and phase respectively. In the analytic signal all negative frequencies have been removed. It can be demonstrated that the modulus $A(t)$ of the analytic signal,

$$
A(t)=\sqrt{x^{2}(t)+\mathscr{X}^{2}(t)},
$$

represents the envelope of the original real-valued signal $x(t)$ (see for example Papoulis 1962; Hahn 1996; Bracewell 2000; Papoulis \& Pillai 2002; Mathis, Hutchins \& Marusic 2007). As will be seen, this relationship is very useful when attempting to interpret (or demodulate) amplitude-modulated signals. A brief tutorial on the Hilbert transform, with emphasis on physical interpretation, can be found in Bendat \& Piersol (1986).

Amplitude modulation refers to the modulation of a high-frequency signal (carrier signal) with a low-frequency component (modulating signal). The principle is simple: the carrier signal is multiplied by the modulating signal added to a judiciously selected offset $B$. This is well illustrated if one considers a pure harmonic, with the carrier signal defined as

$$
c(t)=C \sin \left(\omega_{c} t+\phi_{c}\right)
$$

where $C$ and $\phi_{c}$ are arbitrary constants. Here, for simplicity, these constants are set to the values 1 and 0 respectively. Figure 3(a) shows a carrier signal with $\omega_{c}=10$. Let us also consider an arbitrary waveform representing the modulating signal

$$
m(t)=M \sin \left(\omega_{m} t+\phi_{m}\right)
$$

where $M$ and $\phi_{m}$ are again arbitrary constants also set to 1 and 0 for simplicity (typically $\omega_{m}<\omega_{c}$; figure $3 b$ shows an example modulating signal with $\omega_{m}=2$ ). 
Amplitude modulation is attained by forming the product

$$
\begin{aligned}
& u(t)=[B+m(t)] c(t), \\
& u(t)=\left[B+\sin \left(\omega_{m} t\right)\right] \sin \left(\omega_{c} t\right),
\end{aligned}
$$

where $B$ represents the offset (set to 2 for the present example). The modulation depth $M / B$ indicates the extent to which the modulated variable fluctuates around its original level. This must be less than 1 to ensure a pure-amplitude-modulated signal. It can be shown that the modulated signal is composed of three Fourier components, a carrier wave $\left(\omega_{c}\right)$ and two sinusoidal waves (known as sidebands) whose frequencies are above and below the original carrier wave, $\left(\omega_{c} \pm \omega_{m}\right) / 2 \pi$.

The modulus of the analytic signal formed from the Hilbert transformation of $u(t)$ would in this case return the original modulating signal $m(t)$ shifted by the constant $B$ and is thus invaluable in demodulating amplitude-modulated signals. An example of the modulated signal as given by (4.7) is shown in figure 3(c), with a modulation depth of 0.5. The dashed line in figure $3(c)$ shows the envelope as calculated from the Hilbert transform, which in this case exactly matches the modulating signal $m(t)$ with the appropriate applied shift $B$.

From this result, a simple analogy with the results of Hutchins \& Marusic (2007b) can be formulated. If we consider the existence of a modulating effect from the largescale structures imposed on the small scales of the near-wall region, this would imply that the envelope of the high-frequency part of the signal (figure $2 c$ ) must be directly correlated with the low-frequency part of the signal (figure $2 b$ ). We will introduce in the following section the procedure used to highlight the coupling between these different scales.

\section{Decoupling procedure}

The coupling between the low- and high-frequency components of the signal is determined in the following way: In figure 1(b), the inner and outer peaks are clearly separated in wavenumber space. Therefore, a 'reasonable' cutoff length scale for the large-scale motions can be established. (We use $\lambda_{x} / \delta=1$ in accordance with Hutchins \& Marusic 2007b, as shown in §3.2.) The short- and long-wavelength components of the signals are obtained by applying spatial/wavelength filters on the raw fluctuating $u$ velocity signal (table 3 ).

In order to determine the relationship between the large- and small-scale structures contained in any velocity signal, the small-scale component of the signal $\left(u_{S}^{+}\right)$was analysed using the Hilbert transformation. This allows us to extract an envelope $\left(E\left(u_{S}^{+}\right)\right)$representative of any modulating effect (assumed here to be the large-scale component $u_{L}^{+}$). From the very simple amplitude modulation example outlined in $\S 4$, the envelope returned by the Hilbert transform would ideally indicate just the long-wavelength signal of the 'modulator'. However, for the case of the fluctuating turbulent signal shown in figures 2,5 and 6 , this is not strictly the case. The 'carrier' is here defined as the small-scale part of the signal $\left(\lambda_{x}<\delta\right)$, which of course is comprised of a broad range of amplitudes and wavelengths (as opposed to the single sinusoid of the idealized amplitude modulation example given in §4). As such the envelope returned by the Hilbert transformation will track not only the large-scale modulation due to the log-region events but also the small-scale variation in the 'carrier' signal. To remove this effect, we filter the envelope at the same cutoff as the large-scale signal $\left(\lambda_{x} / \delta>1\right)$. Hence, a filtered envelope $\left(E_{L}\left(u_{S}^{+}\right)\right)$describing the modulation of smallscale structures is obtained. It is now possible to compute a meaningful correlation 


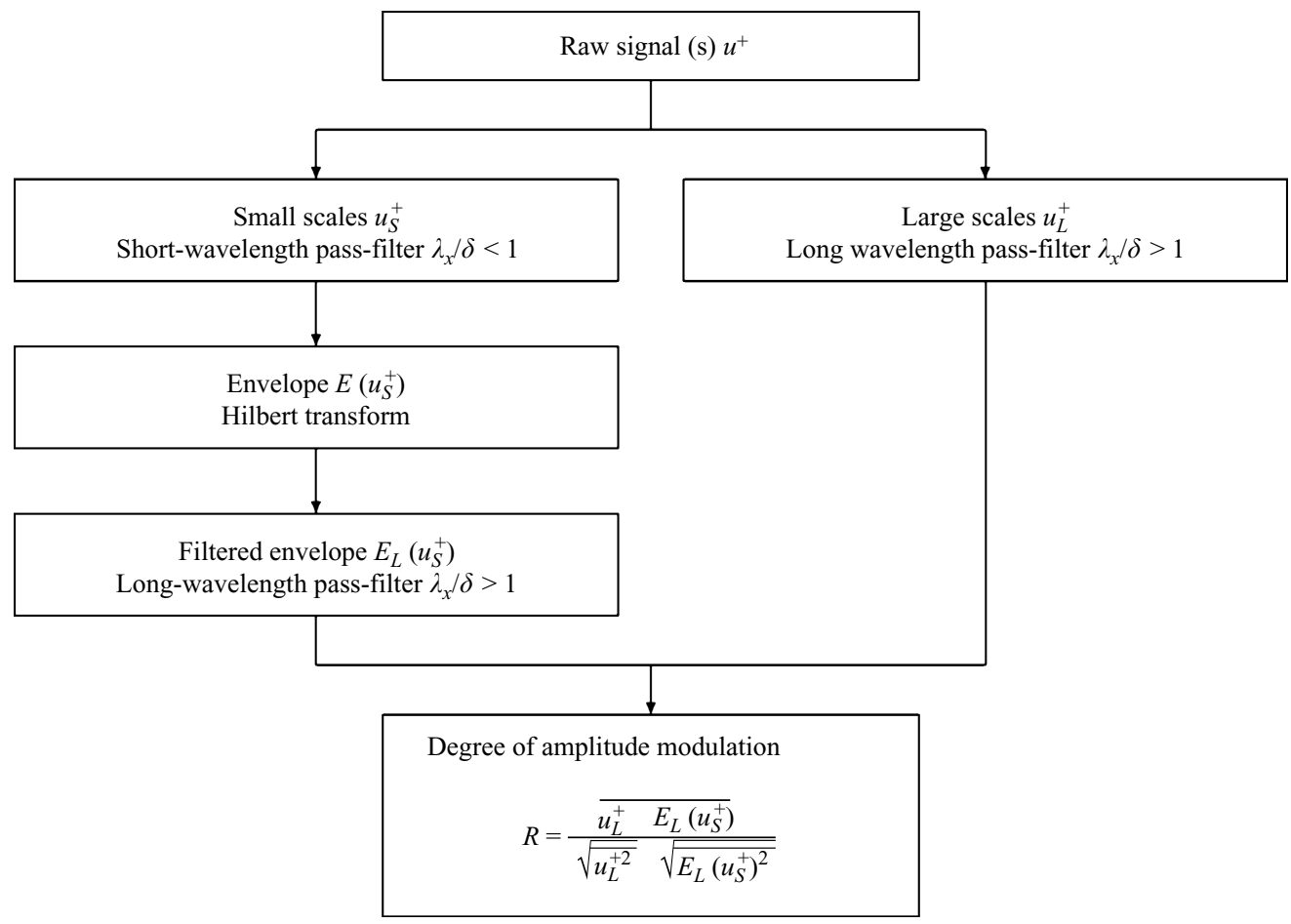

FIGURE 4. Decoupling procedure.

coefficient $R$ of the filtered envelope with the large-scale velocity fluctuation $u_{L}^{+}$:

$$
R=\frac{\overline{u_{L}^{+}} E_{L}\left(u_{S}^{+}\right)}{\sqrt{\overline{u_{L}^{+2}}} \sqrt{\overline{E_{L}\left(u_{S}^{+}\right)^{2}}}},
$$

where $\sqrt{\overline{u^{2}}}$ denotes the root mean square value of the signal $u$. A flow chart of the complete decoupling procedure is given in figure 4 . In the following section, we will show that this procedure is equally applicable to both single-point and twopoint measurements. For two-point measurements, the procedure outlined in figure 4 allows us to estimate the degree to which small scales at any wall-normal location are amplitude-modulated by the large-scale motions from any other wall-normal position.

It should be noted that the procedure employed here is similar to the work of Bandyopadhyay \& Hussain (1984), except that here we use the Hilbert transform to calculate the envelope of the small-scale component.

\section{Example of sample analysis}

The decoupling procedure developed in $\S 5$ is applied here to instantaneous streamwise fluctuating velocity signals at $R e_{\tau}=7300$. Each step of the procedure is demonstrated by showing corresponding example fluctuating signals (large- and small-scale components, envelope and filtered envelope). This affords a clear view of the amplitude modulation effect of the outer-scale events on the near-wall cycle. Since the modulation is mainly assumed to be caused by the action of the large-scale log-region structures on the near-wall small-scale events, the decoupling procedure 


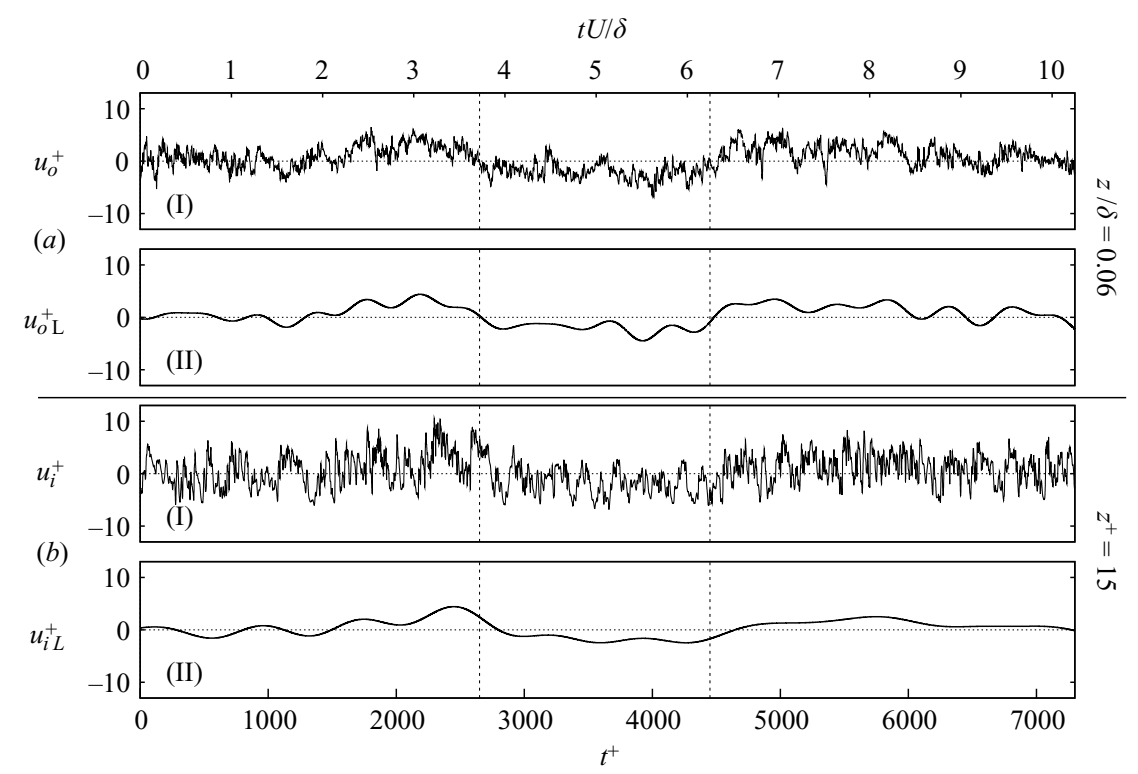

FIGURE 5. Example of large-scale decomposition on the fluctuating $u$ velocity signal: (a) inner-peak location $z^{+}=15 ;(b)$ outer-peak location $z / \delta=0.06$; (I) raw signals, with $37 \%$ of correlation; (II) large-scale components, with $72 \%$ of correlation. Dashed vertical lines show region of negative large-scale $u_{o L}^{+}$fluctuation.

is first applied to the synchronized two-point measurements, located at $z^{+}=15$ and $z / \delta=0.06$ (as described in table 2). Each location represents the peak activity of the two kinds of structures considered in the modulation process: the modulated smallscale motions associated with the inner peak and the modulating superstructure-type events associated with the outer peak. A second analysis is then undertaken on a single-point measurement at $z^{+}=15$. This is to demonstrate that a single-point measurement is sufficient to characterize the degree of amplitude modulation.

\subsection{Two-point analysis}

For the synchronized samples the subscript ' $o$ ' refers to the outer signal and the subscript ' $i$ ' to the inner signal. A time shift of $\Delta T^{+}=90$ is applied to the inner signal, to account for a structural inclination angle of $\theta=14.5^{\circ}$ (this time shift was found to give the maximum correlation), and is consistent with the structure angle reported across many wall-bounded flows by Marusic \& Heuer (2007).

Figure 5 represents raw signals $u_{o}^{+}$and $u_{i}^{+}$(plots I) and their corresponding largescale components $u_{o L}^{+}$and $u_{i L}^{+}$(plots II). A visual comparison of the inner and outer signals indicates a significant degree of correlation. This is confirmed by calculating the long-time average correlations between the signals, found to be $37 \%$ for the raw signals and $72 \%$ for the large-scale components. The high degree of correlation between large-scale components is not surprising. Townsend (1976) already assumed this behaviour through the attached eddy hypothesis, where events close to the wall feel wall-parallel motions due to all attached eddies centred above that height. As shown later by Metzger \& Klewicki (2001) this results in a long-wavelength component superimposed on the near wall. Moreover, Hutchins \& Marusic (2007a) have shown from DNS data $\left(R e_{\tau}=934\right.$; del Álamo et al. 2004) the occurrence in the 


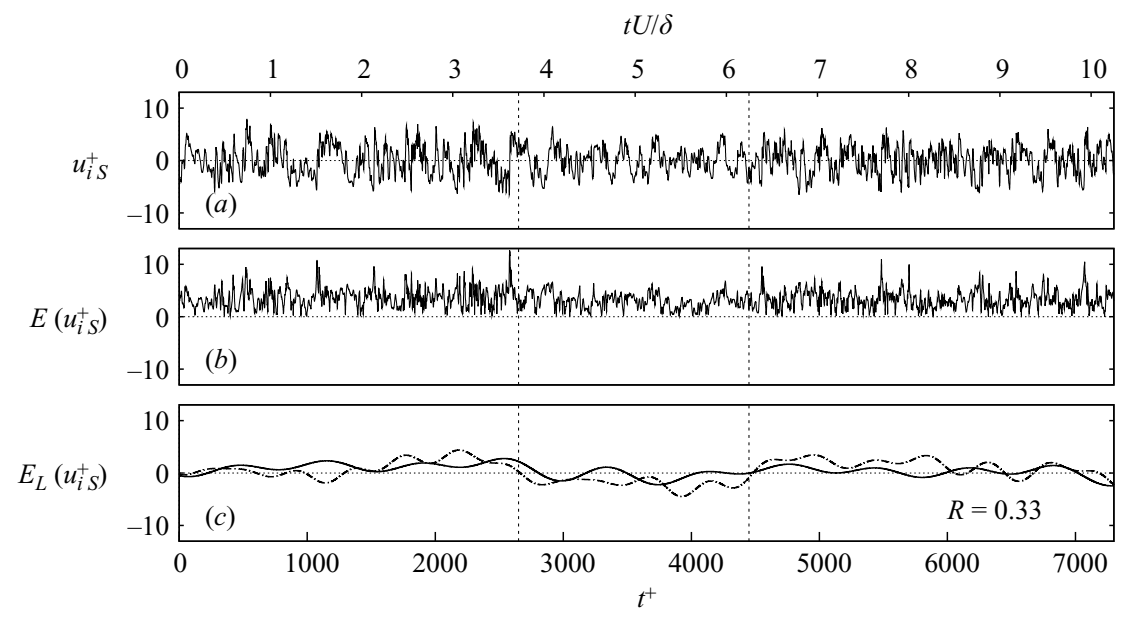

FIGURE 6. Example of small-scale decomposition on fluctuating $u$ velocity signal at $z^{+}=15$ : (a) the small-scale signal $u_{i S}^{+} ;(b)$ its envelope; $(c)$ its filtered envelope (solid line) and the large-scale component at $z / \delta=0.06$ (dot-dashed line). For comparison, the mean of the filtered envelope has been adjusted to zero. Dashed vertical lines show region of negative large-scale $u_{o L}^{+}$fluctuation.

near-wall region of a strong footprint owing to large-scale log-region events, which is basically what we observe here.

The decoupling procedure presented in $\S 5$ is applied here on the inner-signal $u_{i}^{+}$. The only difference is that instead of correlating the filtered envelope with the large-scale component at the same location $\left(u_{i L}^{+}\right)$, we use as a modulating signal the large-scale component at the outer location $\left(u_{o L}^{+}\right)$, which is presumed to be the origin of the modulation. Each step of the decoupling is presented in figure 6 . Figure $6(a)$ shows the short-wavelength pass-filtered inner signal $\left(\lambda_{x} / \delta<1\right)$. This plot shows the modulated small-scale component. Figures $6(b)$ and $6(c)$ show respectively the envelope and the filtered envelope (calculated with the Hilbert transform). The large outer-scale component is superimposed in figure $6(c)$ as a dot-dashed line. (The filtered envelope is amplified by a factor 2 in order to enhance the reading of the figures.) A large-scale low-momentum region is marked by dashed vertical lines. The previously observed attenuation of small-scale near-wall activity within these large-scale low-momentum regions is clearly visible in the small-scale filtered inner signal $u_{i S}^{+}$of figure $6(a)$ and in the Hilbert calculated envelope of figure $6(b)$. The filtered envelope, given in figure $6(c)$, appears to be well correlated to the large outer-scale component, with (5.1) giving $R=0.33$. This represents strong quantitative evidence that the near-wall cycle resides under an amplitude modulation influence imparted by superstructure-type events of the log region.

\subsection{Single-point analysis}

While the two-point synchronized measurements shown above are ideal for studying the modulation influence of the outer-region motions on the near-wall region, these kind of measurements are not easy to perform systematically, and the majority of existing data sets have only single probe traverses across the boundary layer. Thus, a method requiring only single-point measurements would be more immediately applicable and is considered in this section. The main assumption for this analysis is that the footprint at $z^{+}=15$ of the superstructure-type events is sufficient to provide 


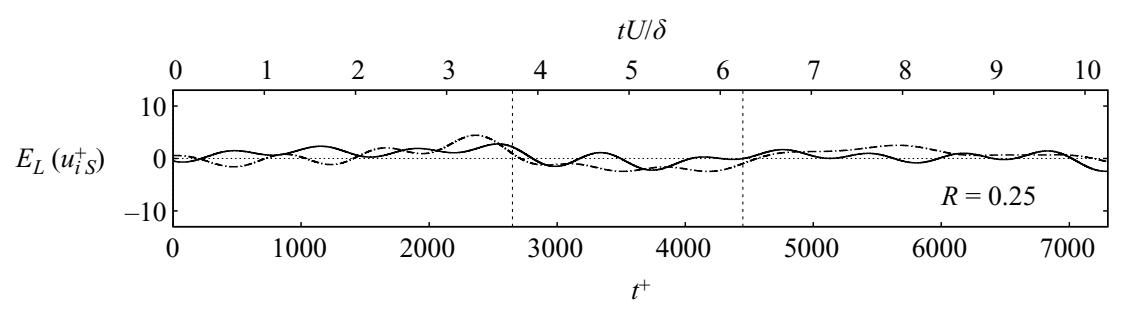

FIGURE 7. Filtered envelope of the small-scale component $E_{L}\left(u_{i S}^{+}\right)$(solid line) at $z^{+}=15$ against its large-scale component $u_{i L}^{+}$(dot-dashed line). For comparison, the mean of the filtered envelope has been adjusted to zero. Dashed vertical lines show region of negative large-scale $u_{o L}^{+}$fluctuation.

a reasonable estimate of the degree of amplitude modulation (without the need for a separate synchronized measurement at the outer peak). This is basically the assumption made in the initial observations of Hutchins \& Marusic (2007a) who analysed only single-point streamwise velocity signals at $z^{+}=15$.

The decoupling procedure applied here is the same as in the previous section, except that the filtered envelope is now correlated to the local large-scale component $u_{i L}^{+}$, which is as originally described by the decoupling procedure shown in figure 4 . Figure 7 shows the filtered enveloped $E_{L}\left(u_{i S}^{+}\right)$against the large-scale component $u_{i L}^{+}$. (Again, the filtered envelope is amplified by a factor 2 in order to enhance the reading of the figures.) The filtered envelope $E_{L}^{+}\left(u_{i S}^{+}\right)$has a significant level of correlation with the large-scale signature of the inner signal $u_{i L}^{+}, R=0.25$. This value is only slightly lower than the one determined using two points (since it comprises only the footprint of the superstructures). Therefore, the single-point analysis seems to provide a reasonable estimate with which to evaluate the degree of modulation and will be used for the analysis of the remaining data at the various Reynolds numbers.

\section{Global evidence of the amplitude modulation effect}

In the previous sections we have considered only the degree of amplitude modulation at the location of the inner peak $z^{+}=15$. To provide more complete evidence of the amplitude modulation effect, the decoupling procedure has been applied at all wall-normal measurement stations across the boundary layer. This results in the correlation coefficient $R\left(z^{+}\right)$, representing the degree of modulation (between the large scales and the filtered envelope) as a function of the wall-normal location. This analysis is applied on the single point traverses described in table 1.

Figure 8 shows the correlation coefficient $R\left(z^{+}\right)$across the boundary layer together with the corresponding spectrogram and mean velocity profile for $R e_{\tau}=7300$. An interesting variation of $R\left(z^{+}\right)$with wall-normal location can be observed. A high level of correlation is observed in the viscous layer of the boundary layer, decreasing progressively towards a plateau occurring between the near-wall region and the log layer. This is interpreted as strong evidence that the near-wall cycle associated with the viscous layer is strongly modulated by low-wavenumber motions associated with the log region. In the log region, the correlation decreases progressively to reach a zero value at about $z^{+} \simeq 300$, corresponding reasonably well to the position of the outer energetic peak as can be seen in figure 8(a). In addition, the zero degree of amplitude modulation as well as the outer peak seem to be located at the approximate mid-point of the $\log$ region. The reversal in correlation behaviour observed in figure $8(b)$ for 


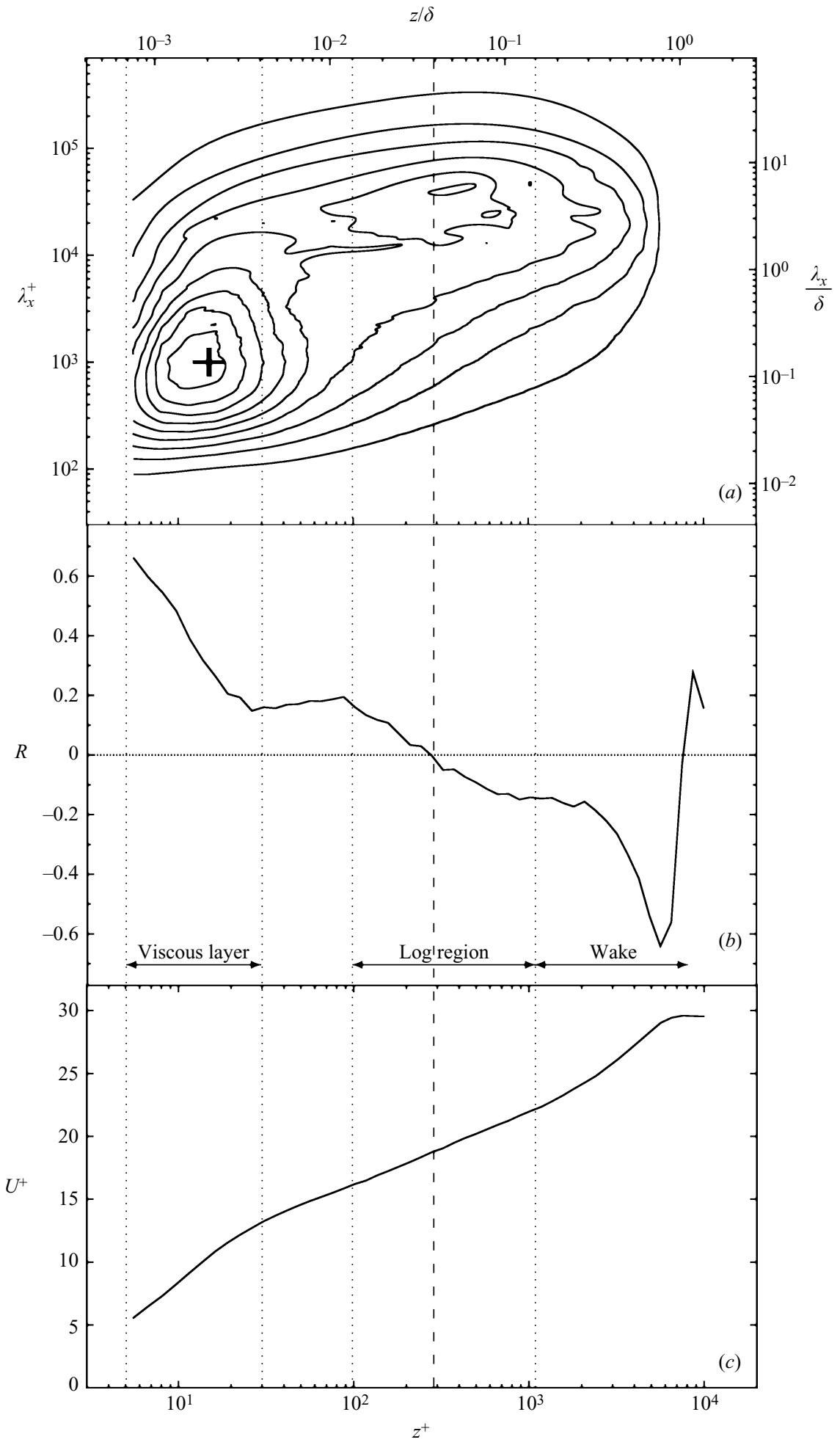

FiguRE 8. Wall-normal evolution of the degree of amplitude modulation: $(a)$ pre-multiplied energy spectra of the streamwise velocity fluctuation $k_{x} \phi_{u u} / U_{\tau}^{2} ;(b)$ correlation coefficient $R\left(z^{+}\right)$ between the large-scale component and the filtered envelope of the small-scale component; (c) mean velocity profile; $R e_{\tau}=7300$. 
higher $z^{+}$is very much as predicted by Hutchins \& Marusic (2007b) who found that the small-scale energy was smaller within large-scale negative fluctuations up to $z^{+} \approx 300$, after which a reversal occurred (and the small-scale fluctuations were more energetic within negative large-scale excursions for $z^{+}>300$ ). At the outermost part of the boundary layer, the high negative peak in the correlation is attributed to intermittency effects. Here the common observation is that the small-scale fluctuations appear only within large-scale signatures (the vast majority of which are negative).

As a side comment, it is interesting to note that the shape of the amplitude modulation correlation coefficient presented in figure $8(b)$ exhibits a strong similarity to the shape of the skewness profile for the streamwise fluctuating velocity. Metzger \& Klewicki (2001) have shown that with increasing Reynolds number the local minima of skewness in the buffer layer move to increasingly positive values. A similar trend is observed for the modulation coefficient $R$. Such similarities are surprising, given that there are clear fundamental differences between skewness, which is a measure of asymmetry in the probability distribution of a signal, and $R$, which is the degree of amplitude modulation between small and large scales.

\subsection{Validation and robustness}

The purpose of this section is to demonstrate that the results presented above are an intrinsic and robust phenomenon of the flow and not mathematical artefacts due to the decoupling procedure. In the first instance, a synthetic signal is studied in order to show that no artificial degree of modulation can result from the different mathematical tools. The subsequent sections are devoted to studying the effect of the cutoff wavelength employed by the filters. This involves an investigation into the effects of the assumed convection velocity (which is used to obtain length scale information from a time signal). A comparison shows that use of local or global convective velocity causes only insignificant changes to the calculated degree of amplitude modulation. Finally, a set of several cutoff wavelengths located between the inner and outer peaks are tested, showing only a weak variation of the degree of amplitude modulation across the boundary layer.

\subsubsection{Synthetic signal}

The synthetic signal is constructed using an original signal at $z^{+}=15$. The original signal has been converted into the spectral domain, using a Fourier transformation, in which the phases are scrambled. (The phase of each Fourier coefficient is replaced with a randomly generated number between 0 and $2 \pi$.) The amplitude remains the same. Figure $9(a)$ shows portions of the original and synthetic signals (left- and righthand plots respectively). Note that from a cursory inspection, both signals look very much like turbulent fluctuating $u$ velocity signals. Figure $9(b)$ shows the corresponding energy spectra for each of the two signals. This technique produces a synthetic signal with exactly the same energy spectra and turbulence intensity as the original signal yet without any realistic phase information. By analysing the filtered signals shown in figure $9(c)$ it is clear that the synthetic signal (right-hand side) does not exhibit any signs of amplitude modulation. Indeed if we compare the negative excursions of the large-scale filtered signal (occurring within the dashed vertical lines) it is clear that the modulating influence on the small-scale $u_{S}^{+}$components, clearly evident for the original signal, is completely absent for the phase-scrambled case. When this analysis is extended to the full signals at all wall-normal locations (figure $9 d$ ), we note that the correlation coefficient $R\left(z^{+}\right)$for the synthetic signal effectively exhibits a zero level across the entire boundary layer. This is in stark contrast to the left-hand plot of 
Original signal

$t U / \delta$
Synthetic signal

$t U / \delta$
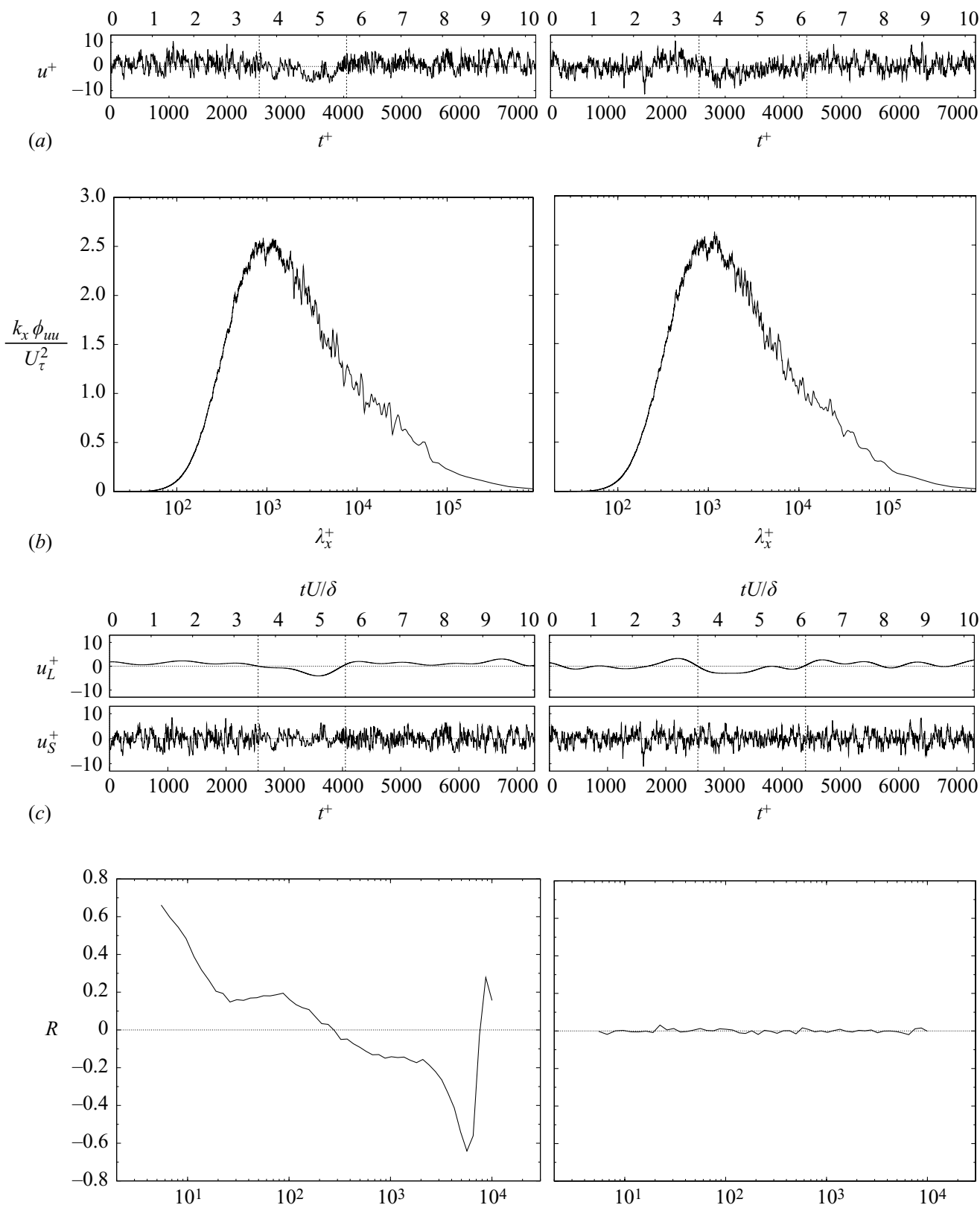

(d)

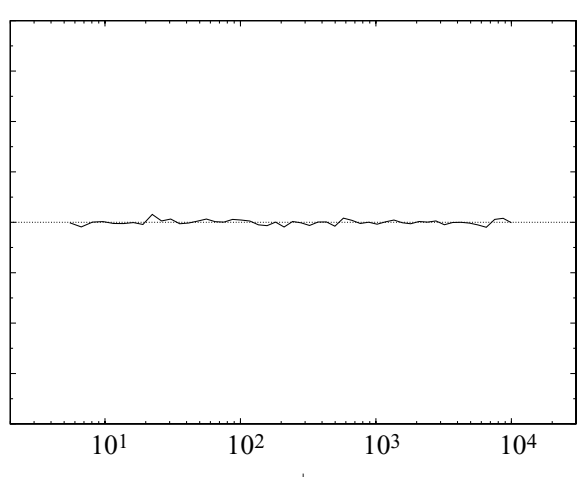

FIGURE 9. Comparison of the decoupling procedure between the original signal (left) and the synthetic phase-scrambled signal (right), at $z^{+}=15, R e_{\tau}=7300:(a)$ instantaneous sample of raw fluctuating signal; $(b)$ pre-multiplied energy spectra; $(c)$ large-scale $\left(u_{L}^{+}\right)$and small-scale $\left(u_{S}^{+}\right)$components (dashed vertical lines on plots $a$ and $c$ show region of negative large-scale fluctuation); $(d)$ correlation coefficient $R\left(z^{+}\right)$between the large-scale component and the filtered envelope of the small-scale component. 

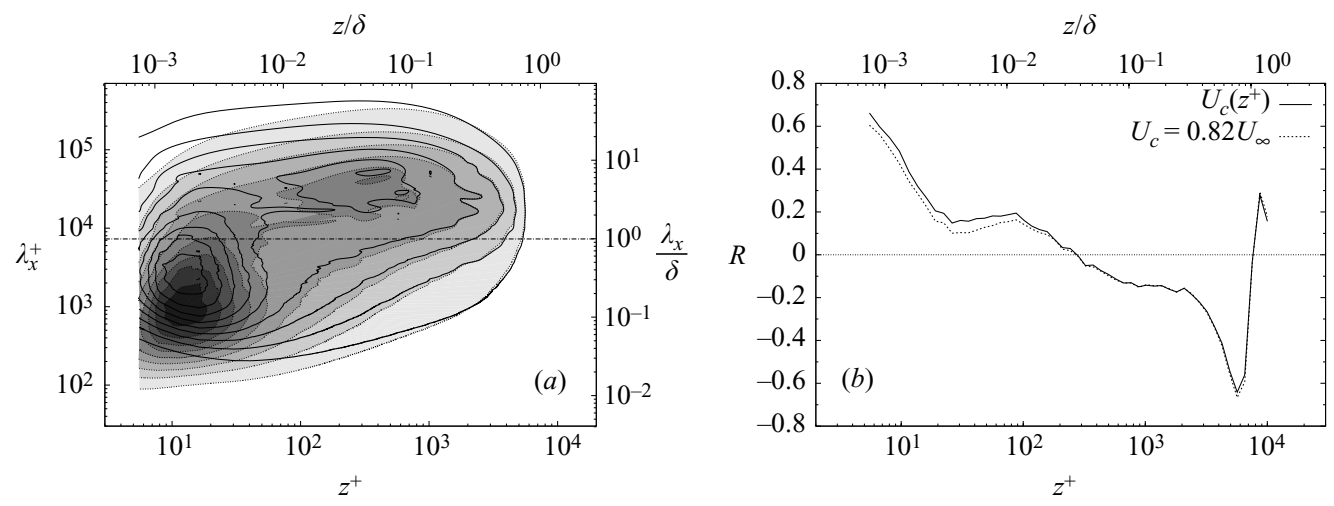

FIGURE 10. Effect of the convection velocity, $R e_{\tau}=7300:(a)$ isocontours of the pre-multiplied energy spectra of streamwise velocity fluctuation $k_{x} \phi_{u u} / U_{\tau}^{2}$, calculated with (shaded contours) the local convective velocity $U_{c}\left(z^{+}\right)$and (solid lines) the global convective velocity $U_{c}=0.82 U_{\infty}$. Contours are from 0.2 to 1.8 in steps of 0.2 . Horizontal dot-dashed lines show the location of the spectral filter. (b) Comparison of correlation coefficient for local (solid) and global (dotted) convection velocity.

figure $9(d)$ (which is reproduced from figure $8 b$ ), which shows that, for the original signal, $R\left(z^{+}\right)$can return high levels of correlation and that it is a strong function of wall-normal position.

\subsubsection{Effect of the convection velocity}

As shown in $\S 5$, the demodulation process is based on spectral filters. Indeed, the first step of the procedure is to decompose the raw signal below and above a cutoff length scale to separate large- and small-scale components. The convection velocity used to construct the pre-multiplied energy-spectra map is thus an important factor, since this determines the energetic length scale $\lambda_{x}^{+}$of the spectra map. Changing the convection velocity will alter the shape of the pre-multiplied energy-spectra map and thus shift the relative position of cutoff wavelengths (perhaps modifying the modulation estimation). Determination of the true convection velocity in turbulent boundary layers remains a controversial subject (Dennis \& Nickels 2008). Regarding it as local (where local convection velocity is determined from the local mean $U_{c}\left(z^{+}\right)=\bar{u}\left(z^{+}\right)$) or global (where the convection velocity is given by some constant across the boundary layer, e.g. $U_{c}=0.82 U_{\infty}$ ) results in changes to the shape of the pre-multiplied energy-spectra map, as shown in figure 10(a). The shaded contours represent the energy spectra obtained using a local convective velocity $U_{c}\left(z^{+}\right)$(used throughout this paper). Solid lines show contours obtained using a global convection velocity $U_{c}=0.82 U_{\infty}$. Significant changes can be observed in wavelength throughout the buffer and log regions, while weak modification appears at the top edge of the boundary layer. Consequently this changes the relative position of the cutoff wavelength, compared to the inner- and outer-peak locations. Indeed, the inner-peak location changes from $\lambda_{x}^{+}=1000$ to $\lambda_{x}^{+} \sim 2200$, while the outer peak is approximately in the same location. However, the cutoff $\left(\lambda_{x} / \delta=1\right)$ still remains located between these two peaks. It is noteworthy that the aim here is not to study the veracity of the local or global convection velocity but to show that whatever pre-multiplied energy-spectra map is constructed, the estimation of the degree of amplitude modulation is only weakly affected. Figure 10(b) compares the degree of amplitude modulation $R\left(z^{+}\right)$ obtained using each convective velocity. The solid line represents the original results 

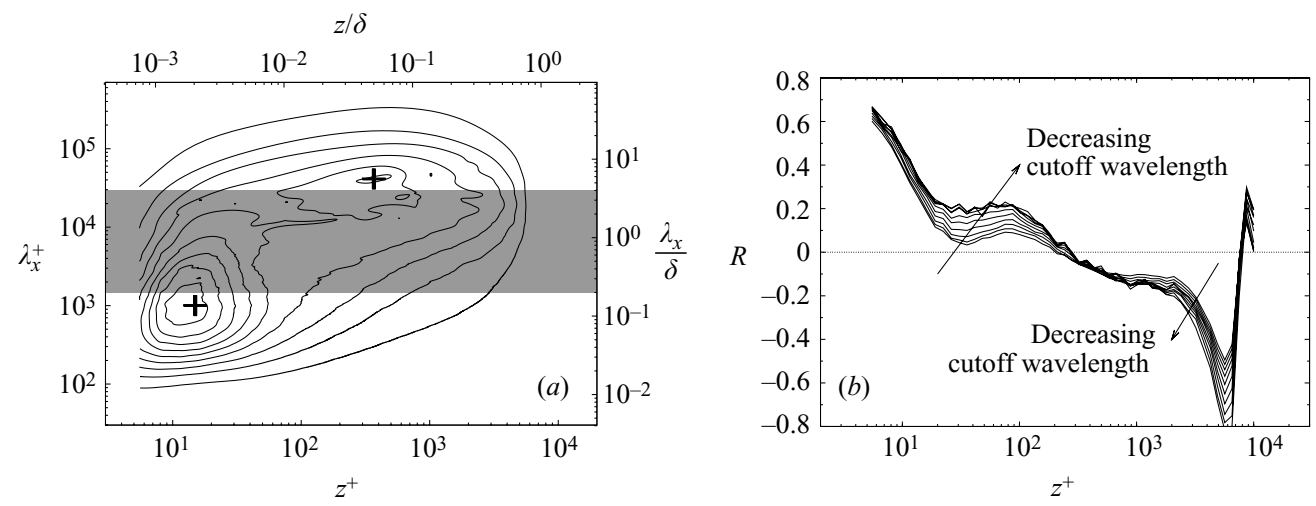

FIGURE 11. Effect of the cutoff wavelength: $(a)$ isocontours of the pre-multiplied energy spectra of streamwise velocity fluctuation $k_{x} \phi_{u u} / U_{\tau}^{2}$ with contours from 0.2 to 1.8 in steps of 0.2 ; shaded area corresponds to the range covered by the set of 10 cutoff wavelengths; the ' + ' symbols mark the inner peak $\left(z^{+}=15, \lambda_{x}^{+}=1000\right)$ and the outer peak $\left(z / \delta=0.06, \lambda_{x} / \delta=6\right)$; (b) comparison of correlation coefficient for different cutoff wavelengths.

obtained using the local convective velocity, whereas the dotted line corresponds to results based on global convection velocity. Except for a weak underestimation of the degree of modulation in the buffer region ( $5 \%$ difference), the wall-normal evolution of the correlation coefficient is in close agreement for both cases. This result shows that the demodulation procedure outlined in figure 4 is only weakly dependent on the choice of convection velocity to build the pre-multiplied energy-spectra map. Furthermore, this analysis would also seem to indicate that the decoupling procedure is somewhat robust to the choice of cutoff wavelength for the filtering. This is explored in more detail below.

\subsubsection{Effect of the cutoff wavelength}

Ten cutoff wavelengths are considered, logarithmically spaced between the inner and outer peaks (from $\lambda_{x} / \delta=0.2$ to $\lambda_{x} / \delta=4$ ). The shaded region in figure 11(a) shows the range of tested cutoff wavelengths on the pre-multiplied energy-spectra map. The demodulation procedure is performed for each cutoff case, and the resulting correlation curves are shown in figure $11(b)$. As the cutoff wavelength is reduced, the magnitude of the measured correlation increases, until a broad maximum is reached at $\lambda_{x} / \delta \approx 0.2$. For even smaller cutoff filters, the magnitude of the correlation will reduce. The general form of the $R\left(z^{+}\right)$results are seen to remain nominally the same and are thus only weakly dependent on the chosen cutoff wavelength. Moreover, the zero value of the amplitude modulation is only minutely affected by the choice of the cutoff wavelengths. This confirms the robustness and the reliability of the decoupling procedure to determine the level of amplitude modulation imparted on the small scale by the large scales.

\section{Reynolds number effects}

It is now well known that the peak of the inner-scaled streamwise turbulence intensity increases with Reynolds number (for example DeGraaff \& Eaton 2000; Metzger \& Klewicki 2001). In particular, it has been shown that this additional energy in the near-wall streamwise fluctuation comes from low-wavenumber events (Metzger \& Klewicki 2001), which we now associate with the large-scaled 

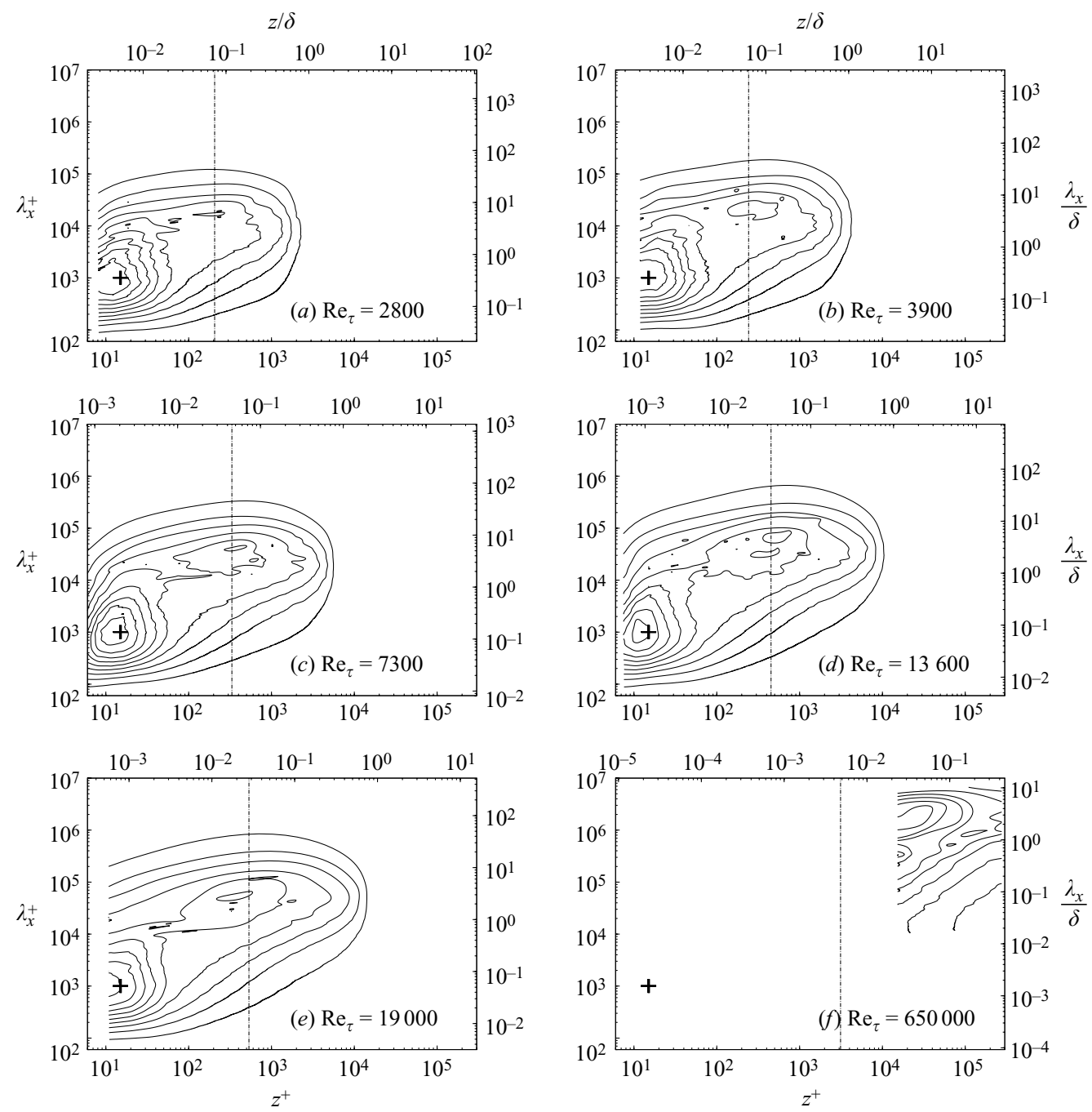

FIGURE 12. Reynolds number effect - iso-contours of the pre-multiplied energy spectra of streamwise velocity fluctuation $k_{x} \phi_{u u} / U_{\tau}^{2}:$ (a) $R e_{\tau}=2800 ;$ (b) $R e_{\tau}=3900 ;(c) R e_{\tau}=7300$; (d) $R e_{\tau}=13$ 600; (e) $R e_{\tau}=19000 ;(f) R e_{\tau}=650000$; Contour levels are form 0.2 to 1.8 in steps of 0.2 ; The large ' + ' marks the inner-peak location $\left(z^{+}=15, \lambda_{x}^{+}=1000\right)$; the vertical dot-dashed line show the middle of the $\log$ layer $\left(3.9 R e_{\tau}^{1 / 2}\right)$.

outer peak. Hutchins \& Marusic (2007a) have shown that the magnitude of the outer peak becomes increasingly comparable to that of the inner peak as Reynolds number increases. Thus, we might expect the modulation caused by the large-scale events on the near-wall cycle to show a similar increase with $R e_{\tau}$. To study this, five sets of hot-wire experimental measurements acquired in the high-Reynolds-number wind tunnel at the University of Melbourne and a set of sonic anemometer measurements from the ASL (SLTEST) are considered. These data cover a range of three decades of $R e_{\tau}$, from 2800 to 650000 . (Details of experimental conditions are given in table 1.)

Figure 12 presents the pre-multiplied energy-spectra maps $k_{x} \phi_{u u} / U_{\tau}^{2}$ for all sets of measurements, in the same manner as presented in figure $1(b)$. The inner peak is marked by the ' + ' symbols and is seen to scale well in viscous units $\left(z^{+}=15\right.$; 

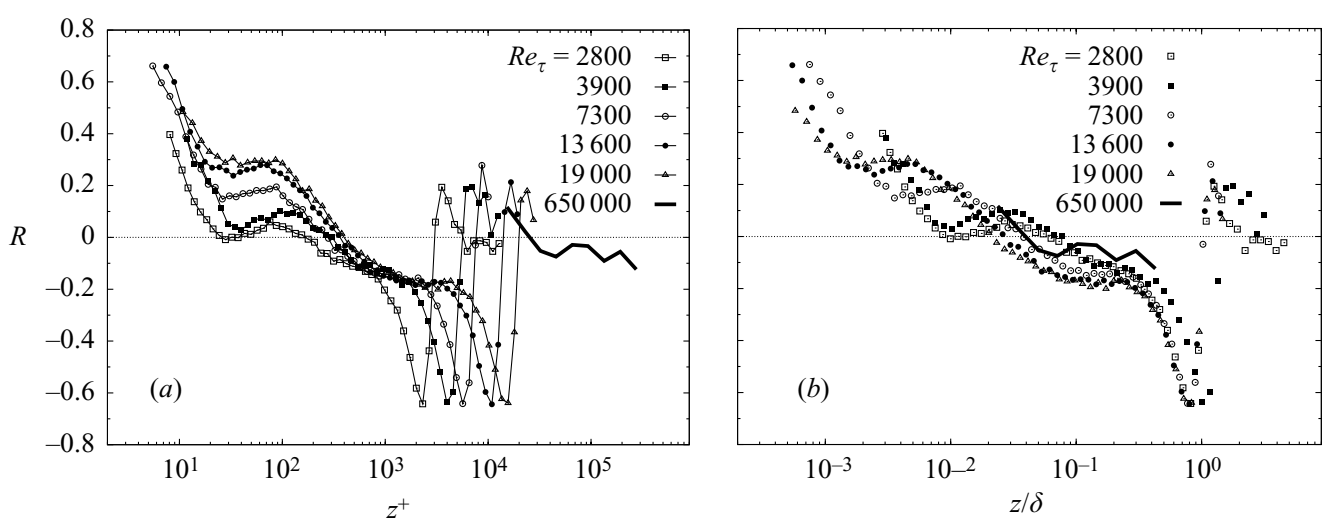

FiguRE 13. Comparison of correlation coefficient between the large-scale component and the filtered envelope of the small-scale component: $(a) R$ plotted in inner-scale $z^{+}$unit; (b) $R$ plotted in outer-scale $z / \delta$ unit.

$\left.\lambda_{x}^{+}=1000\right)$ for all cases in which it has been measured. The location of the outer peak was found by Hutchins \& Marusic (2007a) to be at $z / \delta=0.06$ and $\lambda_{x} / \delta=6$, but this was based on a study over a limited Reynolds number range. Here, the data in figure 12, which cover a larger Reynolds number range, show the location of the outer peak to correspond well with the geometric centre of the logarithmic region (on a log plot), which is indicated by the vertical dashed lines on each plot in figure 12. Over the full range of $R e_{\tau}$ considered, it appears that the cutoff $\lambda_{x} / \delta=1$ remains well located to separate large- and small-scale structures (regarding locations of inner and outer peaks). It should be cautioned that for figure $12(f)$, the data from SLTEST are considerably less reliable than the laboratory data presented in plots $(a)-(e)$. For the SLTEST data, fluctuating signals (and energy spectra) are only available at nine locations within the log region. These fluctuating signals have a limited sample frequency $\left(\Delta T^{+} \approx 100\right)$, and hence the small-wavelength end of the spectra is not captured. In addition, as was previously discussed in $\S 2$, the $1 \mathrm{~h}$ of available data equates to only approximately 350 boundary-layer turnover times. Hence the large-wavelength end of the spectra must be considered improperly converged. Each large-scale 'superstructure' event will take several minutes to advect past the measurement array, and thus the occurrences of these events within the 1 $\mathrm{h}$ of data are limited. In short, figure $12(f)$ should only be considered as indicative of the general form of the spectra at high Reynolds numbers. The apparent peak which is observed in the map at $\lambda_{x} \approx 4 \delta, z^{+} \approx 20000$ is more likely the upper edge of some broadband outer peak. It is noted that in a similar study at SLTEST, Metzger et al. (2007) observed that this peak was approximately located at $z^{+}=2500-3000$ for similar Reynolds number (although these data require the usual caveats with regard to limited convergence, roughness and non-stationarity effects).

To gain a better insight into the $R e_{\tau}$ effects on the amplitude modulation phenomenon, the demodulation procedure has been applied on each data set, at all wall-normal locations across the boundary layer. The resulting $R\left(z^{+}\right)$profiles are respectively plotted in figures $13(a)$ and $13(b)$ in terms of inner scaling and outer scaling. The global shape of each curve is seen to be the same for all $\operatorname{Re}_{\tau}$ (the form of which was analysed in $\S 7$ ). However, certain interesting features can be observed as $R e_{\tau}$ increases. When outer scaled with $z / \delta$, it is interesting to note that figure $13(b)$ exhibits an approximate collapse of the correlation $R(z / \delta)$ over three 


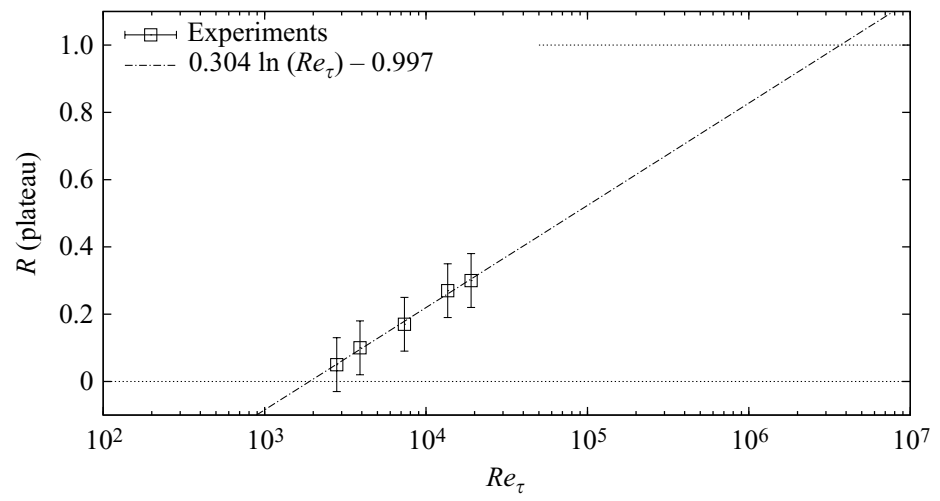

FIGURE 14. Reynolds number evolution of the degree of amplitude modulation in the buffer layer.

orders of magnitude in Reynolds number. This reinforces the assumption that the source of the amplitude modulation mechanism is the large $\delta$-scaled events that inhabit the $\log$ region. In figure 13(a), the degree of amplitude modulation for all Reynolds numbers attains an approximate plateau between the viscous and log regions, $20<z^{+}<100$. The magnitude of this plateau is observed to grow as $R e_{\tau}$ increases. An average value of this plateau is reported in figure 14, which allows us to estimate the Reynolds number dependence of the modulation. The error bars in figure 14 indicate the uncertainty in the plateau magnitude due to choice of cutoff wavelength, as discussed in $\S 7.1 .3$ and as shown in figure $11(b)$. Within these errors, the amplitude modulation effect appears to become significant above $R e_{\tau} \simeq 2000$ which is consistent with observations of Hutchins \& Marusic (2007a) who noted the emergence of the outer energy site at approximately $R e_{\tau}=1900$. Beyond $R e_{\tau}=2000$ the degree of amplitude modulation appears to follow an approximate log law (with $R e_{\tau}$ ). This is consistent with previous work that has shown that the energetic signature of the large-scale motions will increase as compared to the near-wall cycle with increasing Reynolds number. (Hutchins \& Marusic 2007a have shown this increase to be approximately logarithmic with $R e_{\tau}$.) A logarithmic growth of the plateau magnitude is fitted to the data in figure 14 and shown by the dot-dashed line. At very high Reynolds numbers such a growth is unsustainable. Extrapolation of the fit suggests that the correlation would exceed unity when friction Reynolds numbers exceeds $2 \times 10^{6}-4 \times 10^{6}$ (depending on cutoff filter). Since a correlation coefficient cannot exceed unity, a more realistic scenario would be that the magnitude of $R$ could asymptotically approach unity as Reynolds number approaches infinity. This scenario would imply that at very high Reynolds numbers, the near-wall cycle is almost fully driven by the passage of large-scale structures in the log layer. Indeed the basic trend evident in figure 14 would imply that the near-wall cycle at SLTEST will experience a very high degree of amplitude modulation due to the large-scale structures $(R \approx 0.8 \pm 0.05)$.

Another feature of interest is the wall-normal location at which the degree of amplitude modulation crosses zero. This point delineates the wall-normal position at which $R\left(z^{+}\right)$changes sign or to be more precise the location at which the amplitude of the small-scale fluctuations is completely uncorrelated with the large-scale envelope (i.e a position with no amplitude modulation). In figure 8 , for $R e_{\tau}=7300$, the wall-normal position at which $R=0$ (marked with the vertical dashed line) is seen to correspond 

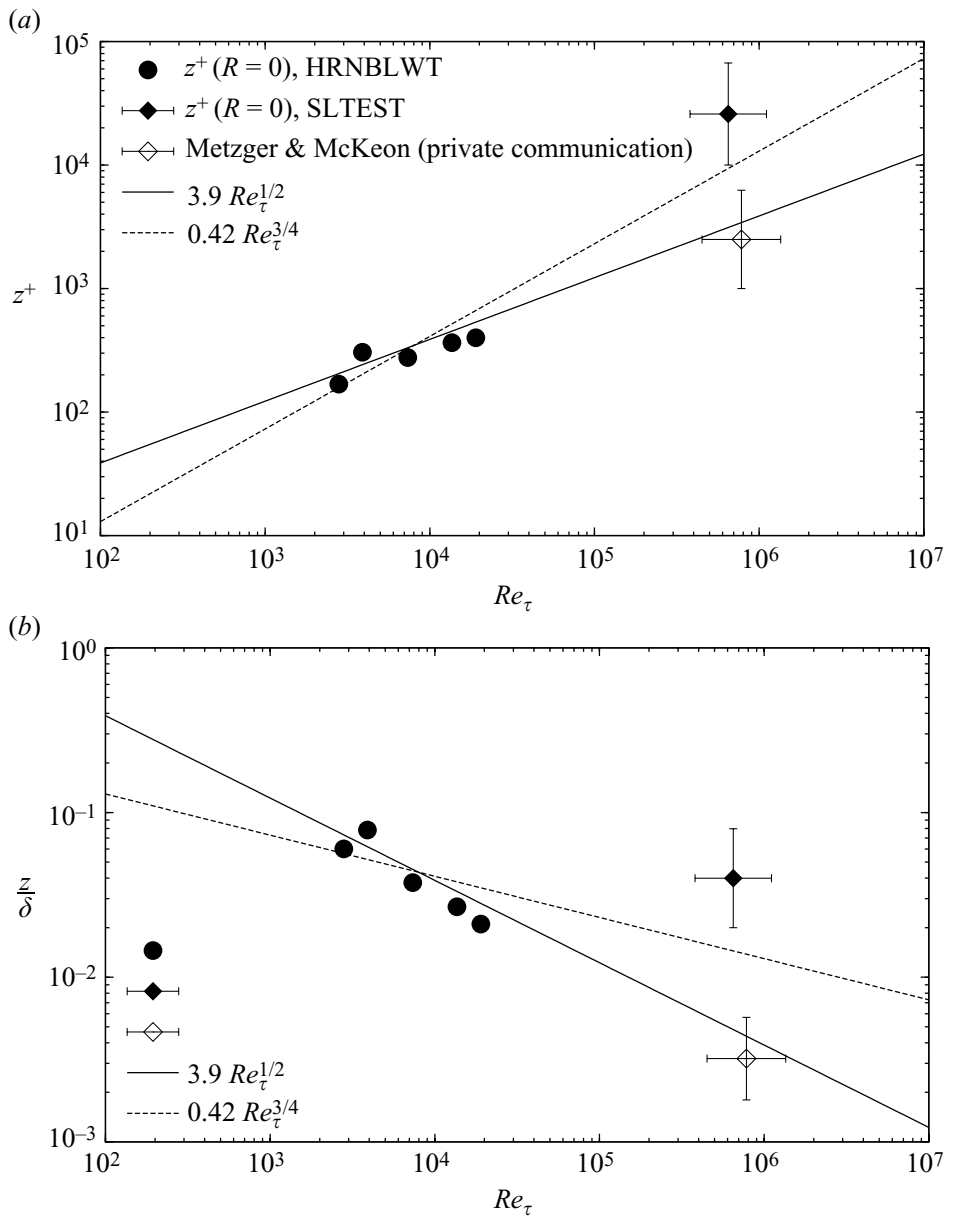

FIGURE 15. Wall-normal location at which the degree of amplitude modulation is zero $(R=0)$ versus Reynolds number (filled symbols): $(a)$ with inner length scaling $\left(z^{+}\right) ;(b)$ with outer length scaling $(z / \delta)$. Lines represent the location of the middle of the log layer, corresponding to (solid) $100<z^{+}<0.15 R e_{\tau}$ and (dashed) based on Reynolds-number-dependent boundaries $K R e_{\tau}^{1 / 2}<z^{+}<0.15 \operatorname{Re}_{\tau}$ (here $K=1.2$ ). The open symbol is the estimated location of the outer $u$-spectral peak measured at SLTEST in 2005 by Metzger \& McKeon (private communication).

well with the location of the outer peak which, as discussed previously, agrees well with the nominal mid-point of the log region. Figure 15 shows as solid symbols the location of the zero amplitude modulation (plotted in inner and outer scalings) for all Reynolds numbers considered here. (It is important to note that the Utah results have large error bars, given the large experimental uncertainties associated with those measurements.) There are considerable differences in the literature as to what constitutes the bounds of the log law. Here, our attention is on a nominal location (rather than a precise one). However, for comparison two lines are shown in figure 15 as estimates of the centre of the logarithmic region (on a log plot). The solid line corresponds to a definition of the $\log$ region as $100<z^{+}<0.15 R e_{\tau}$, giving $z_{M}^{+}=3.9 R e_{\tau}^{1 / 2}$, while the dotted line is based on $K R e_{\tau}^{1 / 2}<z^{+}<0.15 R e_{\tau}$, giving $z_{M}^{+}=0.42 R e_{\tau}^{3 / 4}$, from suggestions by Klewicki et al. (2007) and others that the lower bound of the log region is Reynolds-number-dependent. 
Overall, the results in figures 12 and 15 support the notion that the outer peak in the streamwise energy spectra is coincident with the wall-normal location at which the degree of amplitude modulation crosses zero and that this nominally agrees with the centre of the log region (all of which are Reynolds-number-dependent). It is difficult to interpret this result within a mechanistic description of the boundary layer. Close to the wall, the positive values of correlation are as expected. A large-scale deviation in the local streamwise velocity will produce a corresponding change in the local velocity gradient at the wall, altering the turbulence production (or input of vorticity) in the near-wall region. Thus, in this region the magnitude (or envelope) of the smallscale fluctuations would be expected to follow the sign of the large-scale fluctuations (hence the positive values of $R$ ). Based on the hairpin packet paradigm, one would expect opposite behaviour to occur at some point within the log region. A regime of hairpin packets would imply that most of the small-scale vortical activity would be located within or about the large-scale regions of negative velocity fluctuation (hence the negative values of $R$ in the log region). However, at present we lack an adequate explanation for why the reversal in sign between these two regions should correspond so well to the location of the 'outer site' in the energy spectra.

Also included in figure 15 is an estimate for the location of the outer peak in $u$ spectra as obtained by Metzger \& McKeon (private communication 2009) in separate SLTEST measurements. These data correspond to conditions different from our own and were taken in 2005 with hot wires much closer to the surface - as reported by Metzger et al. (2007) and Guala, Metzger \& McKeon (2009). The error bars for the SLTEST measurements are difficult to estimate, and as such these results should be used with due caution. In any event, the estimate of $z^{+} \approx 2500$ for the outer spectral peak location agrees well with the line indicating the centre of the log region following the $R e_{\tau}^{1 / 2}$ formulation.

The results in figure 15 also have significance in the context of the study of the mean momentum balance by Klewicki et al. (2007) and Fife et al. (2005). From their theory, a line following $z^{+}=C\left(\delta^{+}\right)^{1 / 2}(C=O(1))$ is associated with the location above which viscous force is negligible and below which the viscous force is of the same order as turbulent inertia. (This line tracks the boundary of layers III and IV; see table 2 in Klewicki et al. 2007). Conversely, a line tracking a $3 / 4$ power resides in the middle of the inertially dominated layer IV of the mean momentum balance. This therefore leads to different interpretations of the $R=0$ crossing location (relative to the mean dynamics), depending on whether we consider the data best described by $R e_{\tau}^{1 / 2}$ or $R e_{\tau}^{3 / 4}$. At this stage we consider the question open. Though the present results in figure 15 tend to better support an $R e_{\tau}^{1 / 2}$ trend, there is significant uncertainty in the ASL results. This further highlights the need for high-fidelity measurements in the very high-Reynolds-number range $\left(R e_{\tau} \approx 100000\right)$.

\section{Conclusions}

An analysis of scale-decomposed fluctuating velocity using the Hilbert transformation has revealed strong supporting evidence to confirm the initial assumptions proposed by Hutchins \& Marusic (2007b). In addition to the low-wavenumber energy superimposed on the near-wall streamwise fluctuation (Metzger \& Klewicki 2001), it is found that a nonlinear phenomenon of amplitude modulation takes place between large- and small-scale motions. Indeed, it is shown that in the viscous and buffer layers, the large-scale component is analogous to a modulating signal, while the small-scale component can be viewed as a modulated signal. This apparent 
amplitude modulation, imposed by large-scale log-region events on near-wall viscous-scaled structures, has numerous implications for our assumptions concerning turbulent boundary layers. The near-wall cycle, assumed for some time now to be an autonomous process, is shown here to reside under the modulating influence of the superstructure-type events associated with the log region.

The demodulation procedure built upon the initial observations of Hutchins \& Marusic (2007b) constitutes a valuable tool to analyse the relationship between large- and small-scale structures in turbulent wall-bounded flows. By studying several parameters of the demodulation process, it is shown that the estimation of the amplitude modulation is only weakly affected by possible approximations or errors introduced by the different steps in the demodulation procedure (pre-multiplied energy-spectrum map, convection velocity and spectral filter). This reinforces that the results presented here are robust.

As shown by Hutchins \& Marusic (2007a), the large-scale log-region activity increases in intensity with Reynolds number. This is confirmed by the present study, where an increase of the amplitude modulation is observed as the Reynolds number increases. These experimental data covering three orders of magnitude in Reynolds number have also revealed that the zero magnitude of the modulation collapses well with the mid-point of the log layer, as well as with the location of the outer peak. All of this points towards the conclusion that the large-scale structures play an increasingly important role in high-Reynolds-number turbulent boundary layers. Future efforts will be directed towards using this information for the active control of turbulence, through drag reduction or lift enhancement, at high Reynolds numbers.

We gratefully acknowledge the financial support of the Australian Research Council through grants DP0663499, FF0668703, and DP0984577.

\section{REFERENCES}

Abe, H., Kawamura, H. \& Chol, H. 2004 Very large-scale structures and their effects on the wall shear-stress fluctuations in a turbulent channel flow up to $R e_{\tau}=640$. Trans. ASME, J. Fluid Engng 126, 835-843.

Adrian, R. J., Meinhart, C. D. \& Tomkins, C. D. 2000 Vortex organization in the outer region of the turbulent boundary layer. J. Fluid Mech. 422, 1-54.

Del Álamo, J. C. \& Jiménez, J. 2003 Spectra of the very large anisotropic scales in turbulent channels. Phys. Fluids 15 (6), L41-L44.

del Álamo, J. C., Jiménez, J., Zandonade, P. \& Moser, R. D. 2004 Scaling of the energy spectra of turbulent channels. J. Fluid Mech. 500, 135-144.

Bailey, S. C. C., Hultmark, M., Smits, A. \& Schultz, M. P. 2008 Azimuthal structure of turbulence in high Reynolds number pipe flow. J. Fluid Mech. 615, 121-138.

Bandyopadhyay, P. R. \& Hussain, A. K. M. F. 1984 The coupling between scales in shear flows. Phys. Fluids 27 (9), 2221-2228.

Bendat, J. S. \& Piersol, A. G. 1986 Random Data: Analysis and Measurement Procedure, 2nd edn. Wiley InterScience.

Blackwelder, R. F. \& Kovasznay, L. S. G. 1972 Time scales and correlations in a turbulent boundary layer. Phys. Fluids 15, 1545-1554.

Bracewell, R. 2000 The Fourier Transform and Its Applications, 3rd edn. McGraw-Hill.

DeGraAfF, D. B. \& Eaton, J. K. 2000 Reynolds number scaling of the flat-plate turbulent boundary layer. J. Fluid Mech. 422, 319-346.

Dennis, D. J. C. \& Nickels, T. B. 2008 On the limitations of Taylor's hypothesis in constructing long structures in a turbulent boundary layer. J. Fluid Mech. 614, 197-206.

Fife, P., Wei, T., Klewicki, J. \& McMurtry, P. 2005 Stress gradient balance layers and scale hierarchies in wall bounded turbulent flows. J. Fluid Mech. 532, 165-189. 
Ganapathisubramani, B., Clemens, N. T. \& Dolling, D. S. 2006 Large-scale motions in a supersonic boundary layer. J. Fluid Mech. 556, 271-282.

Ganapathisubramani, B., Longmire, E. K. \& Marusic, I. 2003 Characteristics of vortex packets in turbulent boundary layers. J. Fluid Mech. 478, 35-46.

Guala, M., Hommema, S. E. \& Adrian, R. J. 2006 Large-scale and very-large-scale motions in turbulent pipe flow. J. Fluid Mech. 554, 521-542.

Guala, M., Metzger, M. M. \& McKeon, B. J. 2009 Interactions across the turbulent boundary layer at high Reynolds numbers. In preparation.

Hahn, S. L. 1996 The Hilbert Transforms in Signal Processing. Artech House.

Hambleton, W. T., Hutchins, N. \& Marusic, I. 2006 Simultaneous orthogonal-plane particular image velocimetry measurements in turbulent boundary layer. J. Fluid Mech. 560, 53-64.

HoYAS, S. \& JimÉNEZ, J. 2006 Scaling of the velocity fluctuations in turbulent channels up to $R e_{\tau}=2003$. Phys. Fluids 18, 011702.

Hristov, T., Friehe, C. \& Miller, S. 1998 Wave-coherent fields in air flow over ocean waves: Identification of cooperative behaviour buried in turbulence. Phys. Rev. Lett. 81 (23), 5245 5248.

Huang, N. E., Shen, Z. \& Long, S. R. 1999 A new view of the nonlinear water waves: the Hilbert spectrum. Annu. Rev. of Fluid Mech. 31, 417-457.

Hunt, J. C. R. \& Morrison, J. F. 2000 Eddy structure in turbulent boundary layers. Eur. J. Mech. B 19, 673-694.

Hutchins, N. \& Marusic, I. 2005 Inclined cross-stream stereo particle image velocimetry measurements in turbulent boundary layers. J. Fluid Mech. 541, 21-54.

Hutchins, N. \& Marusic, I. 2007a Evidence of very long meandering features in the logarithmic region of turbulent boundary layers. J. Fluid Mech. 579, 1-28.

Hutchins, N. \& Marusic, I. $2007 b$ Large-scale influences in near-wall turbulence. Phil. Trans. R. Soc. Lond. A 365, 647-664.

Hutchins, N., Nickels, T., Marusic, I. \& Chong, M. S. 2009 Spatial resolution issues in hot-wire anemometry. J. Fluid Mech. In press.

JimÉneZ, J. \& Pinelli, A. 1999 The autonomous cycle of near-wall turbulence. J. Fluid Mech. 389, 335-359.

Jones, M. B., Marusic, I. \& Perry, A. E. 2001 Evolution and structure of sink-flow turbulent boundary layers. J. Fluid Mech. 428, 1-27.

Kim, K. C. \& Adrian, R. J. 1999 Very large-scale motion in the outer layer. Phys. Fluids 11, 417-422.

Klewicki, J. C. \& FAlCO, R. E. 1990 On accurately measuring statistics associated with small-scale structure in turbulent boundary layers using hot-wire probes. J. Fluid Mech. 219, 119-142.

Klewicki, J., Fife, P., Wei, T. \& McMurty, P. 2007 A physical model of the turbulent boundary layer consonant with mean momentum balance structure. Phil. Trans. R. Soc. Lond. A $\mathbf{3 6 5}$, 823-840.

Klewicki, J. C., Metzger, M. M., Kelner, E. \& Thurlow, E. M. 1995 Viscous sublayer flow visualizations at $\operatorname{Re}_{\theta}=1500000$. Phys. Fluids 7, 857-963.

Kline, S. J., Reynolds, W. C., Schraub, F. A. \& Rundstadler, P. W. 1967 The structure of turbulent boundary layers. J. Fluid Mech. 30, 741-773.

KunKel, G. J. \& Marusic, I. 2006 Study of the near-wall-turbulent region of the high-Reynoldsnumber boundary layer using atmospheric flow. J. Fluid Mech. 548, 375-402.

Marusic, I. \& Heuer, W. D. C. 2007 Reynolds number invariance of the structure angle in wall turbulence. Phys. Rev. Lett. 99, 114501.

Marusic, I. \& Hutchins, N. 2008 Study of the log-layer structure in wall turbulence over a very large range of Reynolds number. Flow. Turbul. Combust. 81, 115-130.

Marusic, I. \& Kunkel, G. J. 2003 Streamwise turbulence intensity formulation for flat-plate boundary layers. Phys. Fluids 15, 2461-2464.

Mathis, R., Hutchins, N. \& Marusic, I. 2007 Evidence of large-scale amplitude modulation on the near-wall turbulence. In 16th Australasian Fluid Mechanics Conference, Gold Cost, Australia.

Metzger, M. M. \& Klewicki, J. C. 2001 A comparative study of near-wall turbulence in high and low Reynolds number boundary layers. Phys. Fluids 13, 692-701.

Metzger, M. M., Klewicki, J. C., Bradshaw, K. L. \& Sadr, R. 2001 Scaling the near-wall axial turbulent stress in the zero pressure gradient boundary layer. Phys. Fluids 13 (6), 1819-1821. 
Metzger, M., McKeon, B. J. \& Holmes, H. 2007 The near-neutral atmospheric surface layer: turbulence and non-stationarity. Phil. Trans. R. Soc. Lond. A 365, 859-876.

Monty, J. P., Stewart, J. A., Williams, R. C. \& Chong, M. S. 2007 Large-scale features in turbulent pipe and channel flows. J. Fluid Mech. 589, 147-156.

Nickels, T. B., Marusic, I., Hafez, S. \& Chong, M. S. 2005 Evidence of the $k_{1}^{-1}$ law in highReynolds number turbulent boundary layer. Phys. Rev. Lett. 95, 074501.

Ouergli, A. 2002 Hilbert transform from wavelet analysis to extract the envelope of an atmospheric model: examples. J. Atmos. Ocean. Technol. 19, 1082-1086.

Panton, R. L. 2001 Overview of the self-sustaining mechanisms of wall turbulence. Prog. Aerosp. Sci. 37, 341-383.

Papoulis, A. 1962 The Fourier Integral and Its Applications. McGraw-Hill.

Papoulis, A. \& Pillai, S. U. 2002 Probability, Random Variables and Stochastic Processes. McGrawHill.

Rao, K. N., Narasimha, R. \& Badri Narayanan, M. A. 1971 The 'bursting' phenomena in a turbulent boundary layer. J. Fluid Mech. 48, 339-352.

Schoppa, W. \& Hussain, F. 2002 Coherent structure generation in near-wall turbulence. J. Fluid Mech. 453, 57-108.

Spark, E. H. \& DutTon, J. A. 1972 Phase angle consideration in the modelling of the intermittant turbulence. J. Atmos. Sci. 29.

Sreenivasan, K. R. 1985 On the finite-scale intermittency of turbulence. J. Fluid Mech. 151, 81-103.

TARDU, S. F. 2008 Stochastic synchronization of the near wall turbulence. Phys. Fluids 20, 045105.

TOH, S. \& ITANO, T. 2005 Interaction between a large-scale structure and near-wall structures in channel flow. J. Fluid Mech. 524, 249-262.

Tomkins, C. D. \& Adrian, R. J. 2003 Spanwise structure and scale growth in turbulent boundary layers. J. Fluid Mech. 490, 37-74.

Townsend, A. A. 1976 The Structure of Turbulent Shear Flow, 2nd edn. Cambridge University Press.

WARK, C. E. \& NAGIB, H. M. 1991 Experimental investigation of coherent structures in turbulent boundary layers. J. Fluid Mech. 230, 183-208.

Wark, C. E., NAGUiB, A. M. \& Robinson, S. K. 1991 Scaling of spanwise length scales in a turbulent boundary layer. Paper 91-0235. AIAA. 


\section{University Library}

\section{- M M I N E R VA A gateway to Melbourne's research publications}

Minerva Access is the Institutional Repository of The University of Melbourne

Author/s:

Mathis, R;Hutchins, N;Marusic, I

Title:

Large-scale amplitude modulation of the small-scale structures in turbulent boundary layers

Date:

2009-06-10

Citation:

Mathis, R., Hutchins, N. \& Marusic, I. (2009). Large-scale amplitude modulation of the smallscale structures in turbulent boundary layers. JOURNAL OF FLUID MECHANICS, 628, pp.311-337. https://doi.org/10.1017/S0022112009006946.

Publication Status:

Published

Persistent Link:

http://hdl.handle.net/11343/32979 